\title{
CROSS-CORRELATION WEAK LENSING OF SDSS GALAXY CLUSTERS. I. MEASUREMENTS
}

\author{
Erin S. Sheldon ${ }^{1,2}$, David E. Johnston ${ }^{3,4}$, Ryan Scranton ${ }^{5}$, Benjamin P. Koester ${ }^{6,7}$, Timothy A. McKay Me, $^{8,10}$,

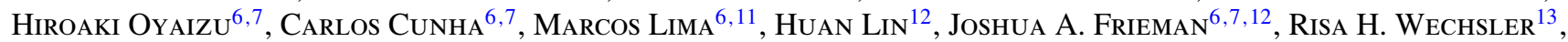 \\ James Annis ${ }^{12}$, Rachel Mandelbaum ${ }^{14}$, Neta A. BahCall ${ }^{15}$, And Masataka Fukugita ${ }^{16}$ \\ ${ }^{1}$ Brookhaven National Laboratory, Upton, New York, NY 11973, USA \\ ${ }^{2}$ Center for Cosmology and Particle Physics, Department of Physics, New York University, 4 Washington Place, New York, NY 10003, USA \\ ${ }^{3}$ Department of Astronomy, 105-24, California Institute of Technology, 1201 East California Boulevard, Pasadena, CA 91125, USA \\ 4 Jet Propulsion Laboratory, 4800 Oak Grove Drive, Pasadena, CA 91109, USA \\ 5 Department of Physics and Astronomy, University of Pittsburgh, 3941 O'Hara Street, Pittsburgh, PA 15260, USA \\ ${ }^{6}$ Kavli Institute for Cosmological Physics, The University of Chicago, 5640 South Ellis Avenue Chicago, IL 60637, USA \\ ${ }^{7}$ Department of Astronomy and Astrophysics, The University of Chicago, 5640 South Ellis Avenue, Chicago, IL 60637, USA \\ ${ }^{8}$ Department of Physics, University of Michigan, 500 East University, Ann Arbor, MI 48109-1120, USA \\ ${ }^{9}$ Department of Astronomy, University of Michigan, 500 Church Street, Ann Arbor, MI 48109-1042, USA \\ ${ }^{10}$ Michigan Center for Theoretical Physics, University of Michigan, 500 Church Street, Ann Arbor, MI 48109-1042, USA \\ ${ }^{11}$ Department of Physics, The University of Chicago, 5640 South Ellis Avenue, Chicago, IL 60637, USA \\ ${ }^{12}$ Fermi National Accelerator Laboratory, P.O. Box 500, Batavia, IL 60510, USA \\ ${ }^{13}$ Kavli Institute for Particle Astrophysics \& Cosmology, Physics Department and Stanford Linear Accelerator Center, \\ Stanford University, Stanford, CA 94305, USA \\ ${ }^{14}$ Department of Physics, Jadwin Hall, Princeton University, Princeton, NJ 08544, USA \\ ${ }^{15}$ Princeton University Observatory, Peyton Hall, Princeton, NJ 08544, USA \\ ${ }^{16}$ Institute for Cosmic Ray Research, University of Tokyo, 5-1-5 Kashiwa, Kashiwa City, Chiba 277-8582, Japan \\ Received 2006 August 25; accepted 2009 August 13; published 2009 September 17
}

\begin{abstract}
This is the first in a series of papers on the weak lensing effect caused by clusters of galaxies in Sloan Digital Sky Survey. The photometrically selected cluster sample, known as MaxBCG, includes $\sim 130,000$ objects between redshift 0.1 and 0.3 , ranging in size from small groups to massive clusters. We split the clusters into bins of richness and luminosity and stack the surface density contrast to produce mean radial profiles. The mean profiles are detected over a range of scales, from the inner halo $\left(25 \mathrm{kpc} h^{-1}\right)$ well into the surrounding large-scale structure $\left(30 \mathrm{Mpc} h^{-1}\right.$ ), with a significance of 15 to 20 in each bin. The signal over this large range of scales is best interpreted in terms of the cluster-mass cross-correlation function. We pay careful attention to sources of systematic error, correcting for them where possible. The resulting signals are calibrated to the $\sim 10 \%$ level, with the dominant remaining uncertainty being the redshift distribution of the background sources. We find that the profiles scale strongly with richness and luminosity. We find that the signal within a given richness bin depends upon luminosity, suggesting that luminosity is more closely correlated with mass than galaxy counts. We split the samples by redshift but detect no significant evolution. The profiles are not well described by power laws. In a subsequent series of papers, we invert the profiles to three-dimensional mass profiles, show that they are well fit by a halo model description, measure mass-to-light ratios, and provide a cosmological interpretation.
\end{abstract}

Key words: dark matter - galaxies: clusters: general - gravitational lensing - large-scale structure of universe

Online-only material: color figures, machine-readable table

\section{INTRODUCTION}

The cold dark matter model (CDM) of structure formation makes a number of predictions about galaxy clusters which are testable observationally. For example, the dark matter in clusters should be more evenly distributed than the observed stars, which are predominantly concentrated in exponential disks and spheroids. The radial distribution of the mass in clusters should, in the mean, follow a predicted universal profile which is a running power law (e.g., Navarro et al. 1997). The number of sub-halos (the centers of which may correspond to galaxies), and the scatter in that number, are predictable as a function of halo mass (Kravtsov et al. 2004). One analytic description of these sub-halo distributions is known as the halo occupation distribution (HOD). The fraction of a cluster's virial mass contained in sub-halos is 10\%-30\% depending on how it is counted (Mao et al. 2004; Gao et al. 2004). The number density of halos as a function of mass has a well-defined form (Press $\&$ Schechter 1974; Sheth \& Tormen 1999; Jenkins et al. 2001).
The cluster two-point correlation and the cluster-mass crosscorrelation function are also predictable in the CDM framework (Mo \& White 1996; Seljak \& Warren 2004); on large scales, they are proportional to the autocorrelation function of the mass, which is given by linear perturbation theory. Each of these predictions depends more or less on the underlying cosmological model.

In the real world, it is difficult to observationally select clusters based upon their mass. Instead, we select clusters based upon some observable and try to relate that observable to mass. For example, a HOD-type description, which uses sub-halos as the basic constituent, can be tested by counting the number (and the dispersion in the number) of constituent galaxies as a function of cluster mass. The key difficulties are estimating accurate cluster masses and relating sub-halos to galaxies. One traditional measure of mass treats the galaxies as tracers of the gravitational potential. For example, in a galaxy redshift survey, one can measure cluster velocity dispersions and, with additional assumptions about the velocity distribution of the 
galaxies relative to the dark matter, infer cluster virial masses (e.g., Carlberg et al. 1996). One can attempt to relate sub-halos to galaxies by matching the observed abundance of galaxies or clusters to the predicted abundance of halos or sub-halos.

With X-ray data, we can use the density and temperature structure of the baryonic gas, with physical assumptions about the state of the system, to infer cluster masses (Henry et al. 1992; Ebeling et al. 1998; Böhringer et al. 2001). In this case, one could in principle bypass the galaxies entirely and use X-ray data alone to infer, for example, the mass function (Reiprich \& Böhringer 2002) or the large-scale clustering (Collins et al. 2000) of X-ray emitters as a function of their mean mass.

While useful, dynamical techniques such as galaxy velocity dispersions and X-ray gas measurements provide limited information on cluster mass profiles, especially on large scales. Galaxy velocity measurements provide only sparse sampling of the cluster potential, although stacking velocity measurements from multiple clusters can yield more precise statistical mass profiles (Mahdavi \& Geller 2004; Katgert et al. 2004; Becker et al. 2007). Moreover, both velocity and X-ray inferences of cluster masses assume that clusters are dynamically simple systems; this assumption may not be justified in general at the requisite level of precision, and there are certainly well-known exceptions to it, such as Coma (Neumann et al. 2003). On scales close to and certainly beyond the virial radius, one expects the assumption of dynamical equilibrium to break down, requiring alternative techniques to estimate the associated mass.

Weak gravitational lensing is, in principle, well suited for studying mass profiles. The first weak lensing detection was in a cluster (Tyson et al. 1990), and the field blossomed rapidly (Fahlman et al. 1994; Tyson \& Fischer 1995; Luppino \& Kaiser 1997; Fischer \& Tyson 1997; Hoekstra et al. 1998; Joffre et al. 2000; Clowe et al. 2000; Dahle et al. 2002; Wittman et al. 2003; Umetsu et al. 2005; Clowe et al. 2006). The lensing effect is sensitive to all mass associated with the cluster, and the interpretation of the shear in terms of mass is independent of the dynamical state of that mass. If the data permit, mass measurements may be extended to very large scales, well beyond the virial radius. However, except in the rare cases where the lensing is very strong, the shape of an individual galaxy gives a very imprecise measurement of the shear, due to the large variety of intrinsic source galaxy shapes. Instead, the shapes of many sources are averaged to increase the sensitivity.

For weak lensing measurements, there are additional sources of error that are not predictable or measurable on a cluster-bycluster basis. Lensing due to structures along the line of sight to the source galaxies, such as voids or distant clusters, can swamp the statistical measurement error (White et al. 2002; Hoekstra 2003). Second, clusters are spatially correlated with other clusters, groups, and galaxies. These associated structures boost the measured lensing signal (e.g., Metzler et al. 2001; White et al. 2002). This increased signal can be significant at almost any point in an individual cluster, and is generally dominant for the average cluster at scales larger than a few virial radii. It is difficult, in general, to identify and model these effects for an individual cluster, which makes recovery of the bound mass uncertain.

The approach we use in this work is a compromise: we average, or "stack," the lensing signal from an ensemble of clusters. In doing so, we cannot recover detailed information about each cluster. This is not a significant sacrifice because the information for individual clusters is not recoverable with high precision due to the sources of error mentioned above. The gains from this technique, however, are significant. The noise due to distant structures along the line of sight, uncorrelated with the lensing cluster, is negligible in the mean under the assumption that the universe is homogeneous and isotropic. The statistical signal from correlated nearby structures, on the other hand, can be modeled using CDM; it dominates on large scales but is typically small within the virial radius.

Another advantage of stacking is that the average cluster mass profile, in the absence of significant selection effects, must be smooth and spherically symmetric if the universe is homogeneous and isotropic. In this case, the lensing measurement, which is related to density in a nonlocal way, can be inverted directly to the average three-dimensional mass profile modulo the mean density of the universe (Johnston et al. 2007a).

The mean cluster mass profile is best interpreted as the crosscorrelation function between clusters and mass. On small scales, this is most sensitive to the mean density profile of the cluster dark matter halos, while on large scales it essentially measures how clusters are correlated with the large-scale structure. For a large enough dynamic range in scale these measurements directly connect the well understood linear growth of perturbations on large scales to the nonlinear collapse of dark matter halos on smaller scales. These ideas have been discussed and verified in simulations in Johnston et al. (2007a), and used to reconstruct the galaxy-mass correlation function in Sheldon et al. (2004).

There is a small literature on ensemble group and cluster lensing. These studies have focused mainly on mass-to-light ratios and cosmology (Hoekstra et al. 2001; Parker et al. 2005). In the Sheldon et al. (2001) pilot study, we studied 42 Sloan Digital Sky Survey (SDSS) clusters matched to X-ray sources in the Rosat All Sky Survey, and demonstrated the feasibility of ensemble cluster lensing in the SDSS.

This work is the first in a series of papers on statistical cluster lensing in SDSS. The clusters used in this study are drawn from a superset of the recently released MaxBCG catalog of Koester et al. (2007a, 2007b), extending that catalog to lower richness objects. We present lensing measurements in bins of cluster richness and cluster $i$-band luminosity, detailed descriptions of our methods, and tests and corrections for systematics. We also present some basic statistics about these profiles, such as tests for redshift dependent signals and comparisons with power-law models (which are not a good description). The idea is to present basic and stable results that will not depend on models of the moment or assumptions about the selection function. This paper is accompanied by Johnston et al. (2007b) in which we present detailed analysis and modeling of the profiles, such as nonparametric inversions of the lensing profiles to three-dimensional density and aperture mass. In that paper, we also model these profiles to extract cluster virial masses and concentrations, large-scale cluster-mass cross-correlations and bias, and the mass-observable relations such as $M-L$ and $M-N_{\text {gals. }}$ This paper is also accompanied by Sheldon et al. (2007), which focuses on cluster mass-to-light ratios. A paper on the mass function and cosmology is also in press (Rozo et al. 2009). We assume that the universe is described by a FriedmanRobertson-Walker cosmology with $\Omega_{M}=0.27, \Omega_{\Lambda}=0.73$, and $\mathrm{H}_{0}=100 \mathrm{~h} \mathrm{~km} \mathrm{~s}^{-1} \mathrm{Mpc}^{-1}$. All distances are measured in physical, or proper, coordinates, not comoving. The basic measures of richness and luminosity we will refer to as $N_{200}$ and $L_{200}$. These are the counts and $i$-band luminosity for galaxies with $L_{i}>0.4 L_{*}$, colors consistent with the cluster ridgeline, and projected separation less than $r_{200}^{\text {gals }}$. See Section 3.5 for more details. 


\section{LENSING FORMALISM AND INVERSIONS}

This section includes a brief description of the lensing formalism used in this paper. More details can be found in Sheldon et al. (2004).

Gravitational lensing is the apparent bending of light as it passes massive objects. The actual path of light is not generally observable, but the distortions produced in the images of sources are. Any distortion produces correlations in the shapes and orientations of background sources, and these correlations are measurable. Note, if some fraction of the sources are in fact not in the background but associated with the lens and if there are "intrinsic alignments" between galaxies in or near the lensing cluster, then the lensing signal will be contaminated. We address this issue in more detail in Section 6.

For statistical weak lensing measurements, the basic observable is the tangential shear $\gamma_{T}$ which, for small shears, is simply proportional to the change in shape of the galaxy

$$
e_{+}=2 \gamma_{T} \mathcal{R}+e_{+}^{\text {int }}
$$

where $e_{+}$is the measured ellipticity of the galaxy, in the tangential frame of reference, and $e_{+}^{\text {int }}$ is the intrinsic shape of the galaxy; the quantity $\mathcal{R}$ is the "responsivity" or "shear polarizability" of the galaxy. It encodes how strongly the image responds to an applied shear, and is measurable in the mean from the ensemble of galaxy shapes (Bernstein \& Jarvis 2002). The intrinsic shape $e_{+}^{\text {int }}$ is the primary source of noise, and its properly weighted rms value is known as the "shape noise," $\sigma_{\mathrm{SN}}^{2}=\left\langle\left(e_{+}^{\text {int }}\right)^{2}\right\rangle$. In the absence of intrinsic alignments, $e_{+}^{\text {int }}$ has zero mean.

The azimuthally averaged tangential shear is related to the geometry of the lens-source-observer system and the projected mass density of the lens:

$$
\gamma_{T}(R) \times \Sigma_{\text {crit }}=\bar{\Sigma}(<R)-\bar{\Sigma}(R) \equiv \Delta \Sigma,
$$

where $\bar{\Sigma}(<R)$ is the mean projected mass density within the disk of transverse radius $R$, and $\bar{\Sigma}(R)$ is the mean within the annulus used to measure the shear. The proportionality, $\Sigma_{\text {crit }}$, encodes the geometry of the lens-source-observer system:

$$
\Sigma_{\text {crit }}^{-1}=\frac{4 \pi G D_{L S} D_{L}}{c^{2} D_{S}},
$$

where $D_{j}$ are the angular diameter distances to lens, source, and between lens and source. Note, the shear measured at $45^{\circ}$ relative to the tangential, $\gamma_{\times}$, should be zero if the signal is due to lensing. As will be discussed in later sections, we have excellent photometric redshifts for each of the cluster lenses, as well as photometric redshifts for each source galaxy; together these provide an estimate of $\Sigma_{\text {crit }}$ and allow us to convert the tangential shear to a measurement of $\Delta \Sigma$.

The signal-to-noise ratio $(\mathrm{S} / \mathrm{N})$ in $\Delta \Sigma$ for a typical lens in the SDSS is much less than unity due to the low redshift of the lenses and sources and the relatively low number density of the source catalog. To increase the sensitivity, we average the $\Delta \Sigma$ measurements from an ensemble of lenses of similar optical properties. This mean signal, measured as a function of projected separation, $R$, is related to the cross-correlation between the lenses and the density field. Under the assumption of statistical isotropy (after stacking, the lenses look spherically symmetric), the mean $\Delta \Sigma$ profile can be inverted to the threedimensional excess density profile:

$$
-\frac{d \Sigma}{d R}=\frac{d \Delta \Sigma}{d R}+2 \frac{\Delta \Sigma}{R}
$$

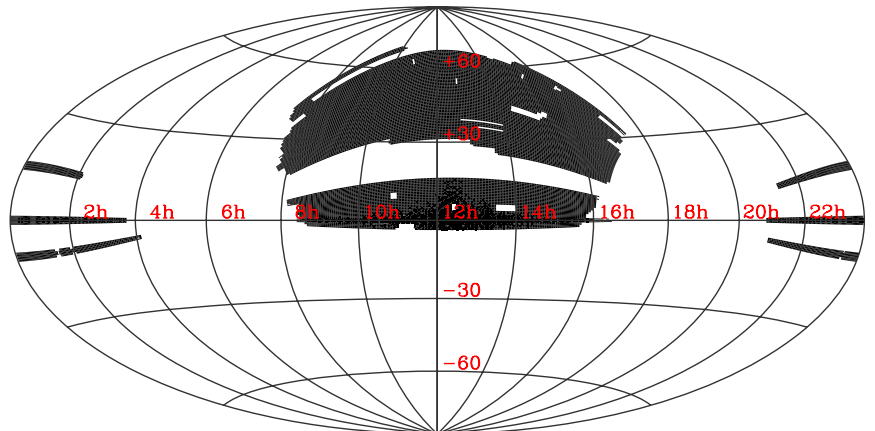

Figure 1. Hammer-Aitoff projection of the sky, with the area used for this analysis shaded in black. This is a subset of the SDSS DR4.

(A color version of this figure is available in the online journal.)

$$
\Delta \rho(r) \equiv \rho(r)-\bar{\rho}=\frac{1}{\pi} \int_{r}^{\infty} d R \frac{-d \Sigma / d R}{\sqrt{R^{2}-r^{2}}}
$$

where $\Delta \rho$ is the mean excess density, relative to the mean density of the universe $\bar{\rho}$. The mean density of the universe will not contribute to the shear in Equation (2), and thus does not contribute to $\Delta \Sigma$ or $\Delta \rho$. This inversion technique was used in Sheldon et al. (2004), and the details are presented in full in Johnston et al. (2007a). The integral in Equation (4) cannot be taken to infinity; in practice this limits the useful range of radii to about $2 / 3$ of the largest radius where measurements are available. As shown in Johnston et al. (2007a), the total mass within radius $r$ can also be recovered from $\Delta \rho$ and $\Delta \Sigma$.

The assumption introduced in Equation (4) is that the correlation function is statistically isotropic. This follows from the isotropy of the universe as long as the cluster finder does not introduce a preferred direction. For example, if the MaxBCG cluster finder, described in Section 3.5, preferentially chose structures oriented along the line of sight, this would violate the assumption that the correlation function is isotropic. Tests and predictions of this effect will be presented in Johnston et al. (2007b).

\section{DATA}

The galaxies used for cluster selection and shear measurement were drawn from the SDSS (York et al. 2000). The area used for this study is somewhat smaller than the SDSS data release 4 (Stoughton et al. 2002; Adelman-McCarthy et al. 2006, hereafter DR4). The coverage area is shown in Figure 1.

The SDSS observing mode is time-delay-and-integrate, with the camera reading out at the scan rate, resulting in an effective exposure time of $54 \mathrm{~s}$. The camera layout, described in Gunn et al. (1998, 2006), comprises six columns of five CCDs. Each CCD in a column is covered with one of the five SDSS filters (Fukugita et al. 1996) and objects pass through each different filter in turn, resulting in nearly simultaneous imaging. The gaps between columns are about a CCD width, and the resulting gaps in the imaging data is scanned on an alternate night.

These data are reduced to object lists through a series of calibration (Hogg et al. 2001; Smith et al. 2002; Tucker et al. 2006), astrometric (Pier et al. 2003), and photometric (Lupton et al. 2001, PHOTO) pipelines. The version of PHOTO used for these data is v5_4. Among the parameters for each object are the position (R.A., decl.), various fluxes, and moments of the light distribution used for shear estimation. These same properties for the point-spread function (PSF), measured from bright stars, are interpolated across the image and used for shape corrections 
(Section 4). Only data that pass a series of automated quality assurance tests are included in the final catalog (Ivezić et al. 2004).

\subsection{Masks}

The area used for this study (see Figure 1) was characterized using the SDSSPix pixelization scheme ${ }^{17}$ to define a window, or mask of the available area. This same scheme has been used for other clustering analyses (Scranton et al. 2003, 2005) and lensing analyses (Sheldon et al. 2004). This mask includes survey boundaries and holes in the survey area. We also exclude regions with inferred extinction greater than $0.2 \mathrm{mag}$ in $r$ (according to the Schlegel et al. (1998) dust maps) from the analysis. Only objects that pass this mask were included in the sample; this includes both the lensing source galaxies and the clusters. This is the area shown in Figure 1. This mask was also used for the edge cuts described in Section 3.5.

\subsection{Source Galaxy Selection}

Candidate source galaxies were drawn from the available area described in Section 3.1. Stars were separated from galaxies using the Bayesian method described in Scranton et al. (2002) and Sheldon et al. (2004). In addition, only galaxies whose size was measured to be much larger than the PSF were used. The cut used is the same as that used in Mandelbaum et al. (2006b): the resolution parameter $R$, which is roughly unity minus the square of the ratio of object PSF size to object size, must be greater than $1 / 3$. The combination of these two cuts is quite conservative: tests based on comparisons with the deeper co-added southern stripe indicate that the stellar contamination is less than $1 \%$.

We discarded objects for which the shape measurement did not converge, large measurement errors $\sigma_{e}>0.4$ and the very tail of the ellipticity distribution $e>4$ (the ellipticity can exceed unity after the dilution correction). Only objects with detections in all five SDSS bandpasses were used, since the accuracy of photometric redshifts is significantly reduced otherwise. Finally, only objects brighter than $r=22$ were included. The final catalog contains 27,912,891 galaxies. The distribution of $r$ magnitudes is shown in Figure 2. The gray curve shows the weighted, "effective" number, where the weight is the inverse shear variance, $1 /\left(\sigma_{e}^{2}+\sigma_{\mathrm{SN}}^{2}\right)$. Note there are a fair fraction of galaxies fainter than 21.5 , but these get little weight in the final analysis.

\subsection{Shape Measurement and Correction}

The details of the shape measurement were given in Sheldon et al. (2004), and are an implementation of the techniques presented in Bernstein \& Jarvis (2002). With this method, the moments of an elliptical Gaussian weight function are matched to those of the object in question through an iterative algorithm. We measured second- and fourth-order moments for all objects in the survey using this method; these parameters are in the SDSS database.

The second order moments $Q_{m, n}$ for each object are combined into the shape parameters,

$$
\begin{array}{r}
Q_{m, n}=\sum_{m, n} I_{m, n} W_{m, n} x_{m} x_{n} \\
e_{1}=\frac{Q_{1,1}-Q_{2,2}}{Q_{1,1}+Q_{2,2}}, \quad e_{2}=\frac{2 Q_{1,2}}{Q_{1,1}+Q_{2,2}},
\end{array}
$$

\footnotetext{
17 http://dls.physics.ucdavis.edu/ scranton/SDSSPix/
}

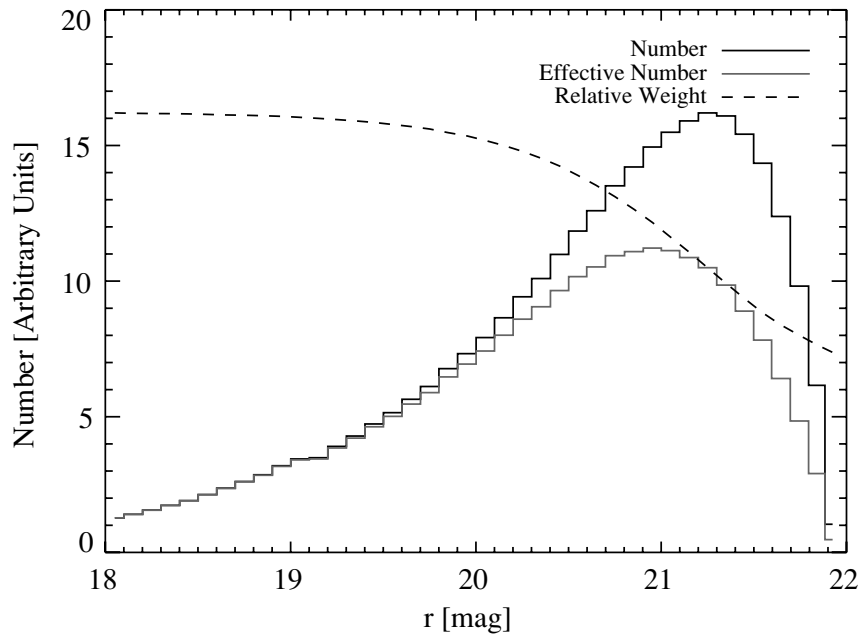

Figure 2. Distribution of source galaxy magnitudes after applying the cuts described in Section 3.2. The black line is the normalized histogram and the gray line is the effective number, normalized to the bright end, after including the weight. The relative weight, shown in arbitrary units as the dashed curve, is an inverse shear variance weight.

where $I_{m, n}$ is the intensity at pixels $m, n, W_{m, n}$ is the radial weight function, and $x_{m}$ are the pixel coordinates relative to the centroid of the galaxy light. These shape parameters are used directly in the shear estimation, as shown in Equation (1). The fourth-order moment accounts for the non-Gaussianity of the object and is part of the resolution parameter, or smear polarizability (Fischer et al. 2000; Bernstein \& Jarvis 2002).

The PSF smears and changes the shape of galaxies, which can be mistaken for the effects of lensing. In order to correct for these effects, the PSF was modeled from bright stars and interpolated to the position of each galaxy using a KL decomposition. This algorithm was described in detail by Lupton et al. (2001) and Sheldon et al. (2004). The effect of the PSF was then corrected for using the techniques of Bernstein \& Jarvis (2002) combined with the "re-Gaussianization" method of Hirata \& Seljak (2003). This method treats the PSF as a Gaussian plus a small nonGaussian component, which is generally a good representation of the SDSS PSF. A convolution kernel is used to transform the image, removing the effect of the non-Gaussian component. The remaining Gaussian PSF component can be corrected for exactly using the formulas in Bernstein \& Jarvis (2002).

These corrections for PSF convolution can be thought of as two separate corrections: the first corrects for the smearing by the finite PSF which makes the object look more round and affects the shear calibration. This is often referred to as PSF dilution. The second alters the shape of the galaxies due to the anisotropy of the PSF. These are often referred to as "multiplicative" and "additive" biases, respectively. In practice, these two biases are coupled.

\subsection{Photometric Redshifts}

The photometric redshifts (photo- $z$ ) were calculated using a neural network based on the training set method of Collister \& Lahav (2004). The spectroscopic training set was collated from the SDSS spectroscopic survey and various surveys with coverage overlapping the SDSS. From a total of $\sim 60,000$ galaxies with measured redshifts, $\sim 45,000$ were from the SDSS main galaxy sample (roughly $r<17.6$; see Strauss et al. 2002), $\sim 14,000$ were from the SDSS luminous red galaxy (LRG) sample (Eisenstein et al. 2001), 1500 were from the CNOC2 


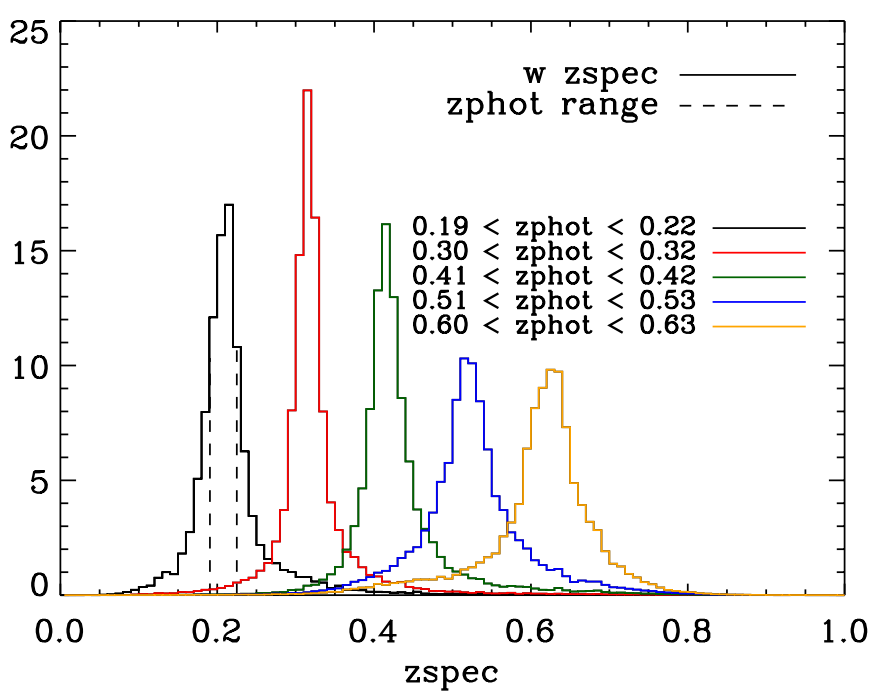

Figure 3. Weighted distribution of spectroscopic redshifts in a few bins of photometric redshifts. Using the technique of Lima et al. (2008), we chose weights such that the weighted magnitude distribution in each of the five SDSS bandpasses matched that of the photometric sample. We then used these weights to generate a weighted redshift distribution that approximates that of the photometric sample. The error bars come from bootstrap re-sampling the spectroscopic validation set.

(A color version of this figure is available in the online journal.)

survey (Yee et al. 2001), and $\sim 300$ were from the CanadaFrance Redshift Survey (Lilly et al. 1995). The training set covers a redshift range between 0 and 1 . Of the $\sim 60,000$ objects, 30,000 were used to train an artificial neural network while the remaining objects (the validation set) were used to check the results of the trained network. The resulting rms scatter in photo$z$ 's for the validation set was $\sim 0.04$ over the entire magnitude range, with more scatter at the faint end. Recall that galaxies with fainter magnitudes get less weight as shown in Figure 2. The details of these methods can be found in Oyaizu et al. (2008).

These redshift estimates were used in the lensing analysis to calculate the signal for each lens-source pair, but the photometric redshifts have remaining errors. We used the validation set to constrain the true redshift distribution in a given bin of photo- $z$ using the weighting technique described in Lima et al. (2008), and then corrected the lensing signal statistically. In this technique, weights are calculated for each spectroscopic galaxy such that the weighted magnitude distribution for the spectroscopic sample matches that of the photometric sample in each of the five SDSS bandpasses. These weights were then used to create a weighted redshift distribtion that should match that of the photometric sample. Lima et al. (2008) found that this method reproduces the underlying distributions with a high level of accuracy in simulations where the only variables for selection were magnitude. In the real data, other factors may be important such as surface brightness and angular size. These factors have not yet been addressed and may lead to additional errors.

Example distributions for a few photo- $z$ bins are shown in Figure 3. The peak of the distributions is relatively unbiased, indicating that the neural network tends to find the maximum likelihood. However, the distributions are broad and even skewed in some cases.

The lensing $\Delta \Sigma$ depends on the distances to lens and source through the inverse critical density. For each lens-source pair, we integrated over the above distributions in redshift to get the expected inverse critical density. The formalism we used was the same as Sheldon et al. (2004), except here we integrated over the full distribution determined as outlined above, whereas in that work we used the error estimates that came out of a chi-squared analysis over galaxy templates. In this work, we applied no additional prior to the distribution. In the end, the results using only these distributions differed from calculations treating the photo-z's as perfect by only a percent.

We also repeated our analysis for sources in each of the redshift ranges shown in Figure 3, as well as for relative redshift thresholds $z_{\text {source }}>z_{\text {lens }}+\delta z$ up to $\delta_{z}=0.2$. Although the results are generally noisier for the subsamples, we saw consistent results for each sample.

\subsection{Cluster Selection}

The MaxBCG algorithm (Koester et al. 2007a, 2007b) was used to identify clusters in SDSS imaging data using three observational properties of rich galaxy clusters. These properties are (1) spatial clustering, (2) clustering in color space (the red sequence), and (3) the presence of a brightest cluster galaxy (BCG) spatially coincident with the approximate center of the cluster. These model components are folded into a redshiftdependent likelihood function, which has built-in predictions for the colors of the red sequence, the color-magnitude properties of the BCG, and the spatial distribution of cluster members (NFW, Navarro et al. 1997). Every object in the input galaxy catalog is then evaluated for its likelihood of being a BCG in an overdensity of bright ( $>0.4 L_{*}$ in $i$ band) red galaxies (within $\pm 2 \sigma$ of the red sequence) at a grid of redshifts. The $\sigma$ in this case is the intrinsic width of the red sequence which is 0.05 in $g-r$, plus the measurement error for each object. A maximum likelihood redshift is determined for each object, and these potential BCGs are ranked by decreasing maximum likelihood. In a manner analogous to the spherical overdensity algorithm employed in $\mathrm{N}$-body simulations, the highest likelihood potential BCG in the survey is deemed a BCG at the center of a cluster. Any lower likelihood objects in the ranked list within a projected $r_{200}^{\text {gals }}$ (see below) and \pm 0.02 in redshift of this first BCG are eliminated as possible cluster centers. The process is repeated for the next object in the ranked list, given that it is not in the exclusion region of the first object. This prescription is iteratively applied to the entire list, thereby generating the final cluster catalog.

The initial parameters in the cluster catalog include a maximum likelihood redshift (between 0.05 and 0.3 ) and an initial richness estimate, $N_{\text {gal }}$ (the number of red sequence galaxies within $1 \mathrm{Mpc}$ ). The sample is well understood in terms of completeness, purity, and photo- $z$ accuracy within the redshift range $[0.1,0.3]$; at lower redshifts the photo- $z$ is less reliable, so these objects are discarded. Over the redshift interval [0.1,0.3], the photo-z's have a small bias $\sim 0.004$ which we correct and scatter that is $\lesssim 0.01$. The sample appears to have a number density that is roughly independent of redshift, although the presence of a supercluster at redshift 0.08 , the so-called Sloan Great Wall, dominates the statistics at low redshift (Gott et al. 2005).

After these initial parameters are determined for each cluster candidate, measurements of cluster richness and redshift are refined. A scaled radius $r_{200}^{\text {gals }}$ is determined from the $N_{\text {gal }}-r_{200}^{\text {gals }}$ relationship measured by Hansen et al. (2005); it is the radius at which the mean luminosity density reaches 200 times the mean value predicted by the luminosity function. The number 


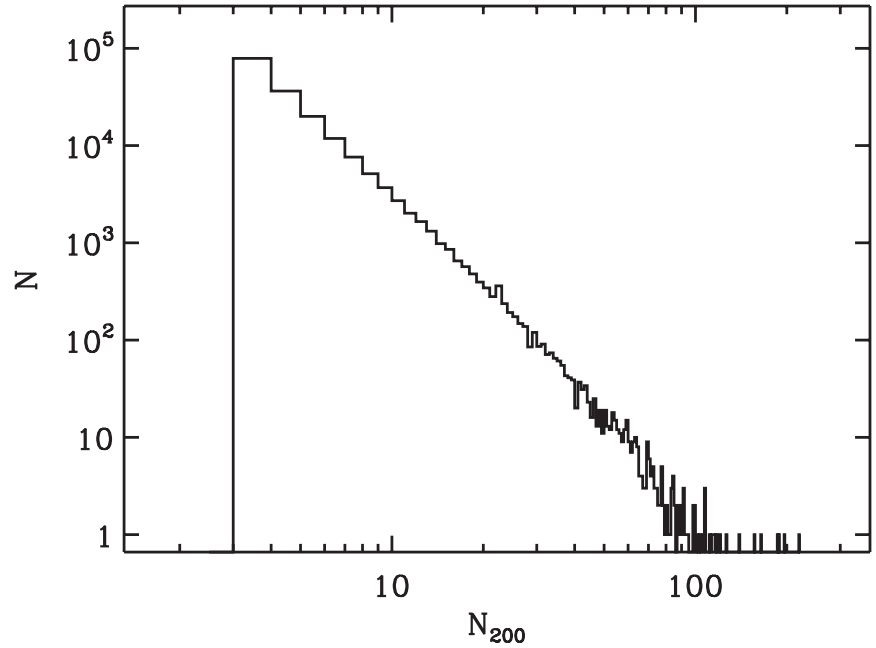

Figure 4. Histogram of $N_{200}$, the number of red galaxies brighter than $0.4 L_{*}$ within $r_{200}^{\text {gals }}$, for the MaxBCG cluster sample.

of galaxies $N_{200}$ and the $i$-band luminosity $L_{200}$ are then recounted within $r_{200}^{\text {gals }}$ with the same selection criteria as above.

Some statistics for the final cluster sample are shown in Figures 4 and 5 The $N_{200}$ function is shown in the left panel. We have extended this sample to $N_{200}=3$, substantially lower than the sample from Koester et al. (2007b). These objects have substantially more noise in their photo- $z$ and richness measures, but as we will see in the following sections they have a well-measured lensing signal. The added noise does, however, complicate the interpretation of the lensing results. In Paper II (Johnston et al. 2007b), we interpret the MaxBCG selection function using the best-available simulations, but a full analysis will require large volume high-resolution simulations that are not currently available. Nevertheless, we present the lensing measurements here in anticipation of future improvements to the simulations.

Figure 5 shows the cluster abundance as a function of redshift. The overall sample is close to volume limited, but the distributions in individual $N_{200}$ bins have significant features in redshift. This is due to some combination of the redshiftdependent model for $0.4 L_{*}$, the definition of the red sequence and true evolution in the number of galaxies at fixed mass and redshift. It is difficult to disentangle these contributions.

Geometrical edge cuts were applied before using the clusters for lensing, in addition to the basic mask cut applied in Section 3.1. As will be explained in Section 4, we require the searchable area surrounding each lens center to be either a full disk or a half disk on the sky. This is to guarantee that there are pairs of sources at $90^{\circ}$ separation with respect to the lens center to cancel residual PSF systematics. We checked each lens against the mask to guarantee this. The maximum search radius is a function of redshift, so lenses at low redshift are more likely to hit an edge. We discarded $21 \%$ of the available clusters leaving 132,473 .

\section{CORRECTIONS TO THE LENSING PROFILES}

Two corrections were made after the basic lensing measurement was complete. The first corrects for the so-called additive bias outlined in Section 3.3, and the second corrects for the clustering of source galaxies with the lenses.

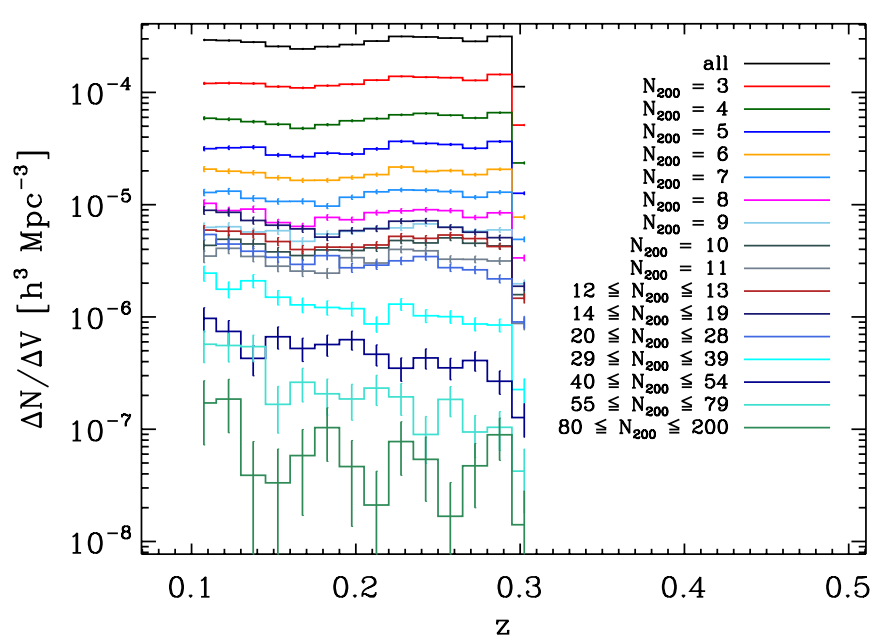

Figure 5. Number density as a function of redshift for differential bins of $N_{200}$. (A color version of this figure is available in the online journal.)

\subsection{Correction for Additive Bias}

The additive bias is due the residual PSF induced ellipticity, left over from imperfect PSF interpolation which results in small inaccuracies in the galaxy shapes after PSF correction. For the most part, this is a random effect across the survey that cancels in the average, but because it is correlated over the scales we are interested in it does not cancel completely. Also, residuals that are constant across the shear measurement area for a given lens will cancel as long as there are pairs at $90^{\circ}$ separation, which prompted the edge cuts outlined in Section 3.5. Components that are not effectively constant, however, may be present. These correlated residuals are confined to an SDSS stripe for the most part, and are thus correlated on angular scales less than a few degrees.

This effect was checked using random points generated over the same area as the lenses and sampling the same systematics. We used the mask generated for this data (see Section 3.1), including the edge cuts applied in Section 3.5. Random points were assigned redshifts such that the redshift histograms of clusters and randoms are proportional when binned at $\Delta z=$ 0.01 . This histogram matching was performed separately for all binnings of the clusters shown below; different samples may have different redshift distributions. This is important because systematics depend mainly on angular scale, which affects different physical scales as a function of redshift.

The results for two sets of random points are shown in Figure 6. The top panel is for $N_{200}=3$, the lowest richness bin, which has the weakest signal. The bottom panel is for a moderately high-richness bin, $12 \leqslant N_{200} \leqslant 17$. On very large scales, the signal for the top panel is strongly affected by this additive systematic, while the signal in the bottom panel is less affected due to the higher signal. The scale of these correlations is a few degree for the mean redshift of 0.25 , as expected. Note, on smaller scales there may be additional correlations that are not detected due to the higher noise, but they are not relevant as the signal from the clusters is much higher. This residual additive bias is subtracted from the lensing signal for all samples presented below. $^{18}$

\footnotetext{
18 Note that this technique of correcting for residual PSF anisotropy using random points only works for lens-shear cross-correlations, not for shear-shear (so-called cosmic shear) correlations.
} 


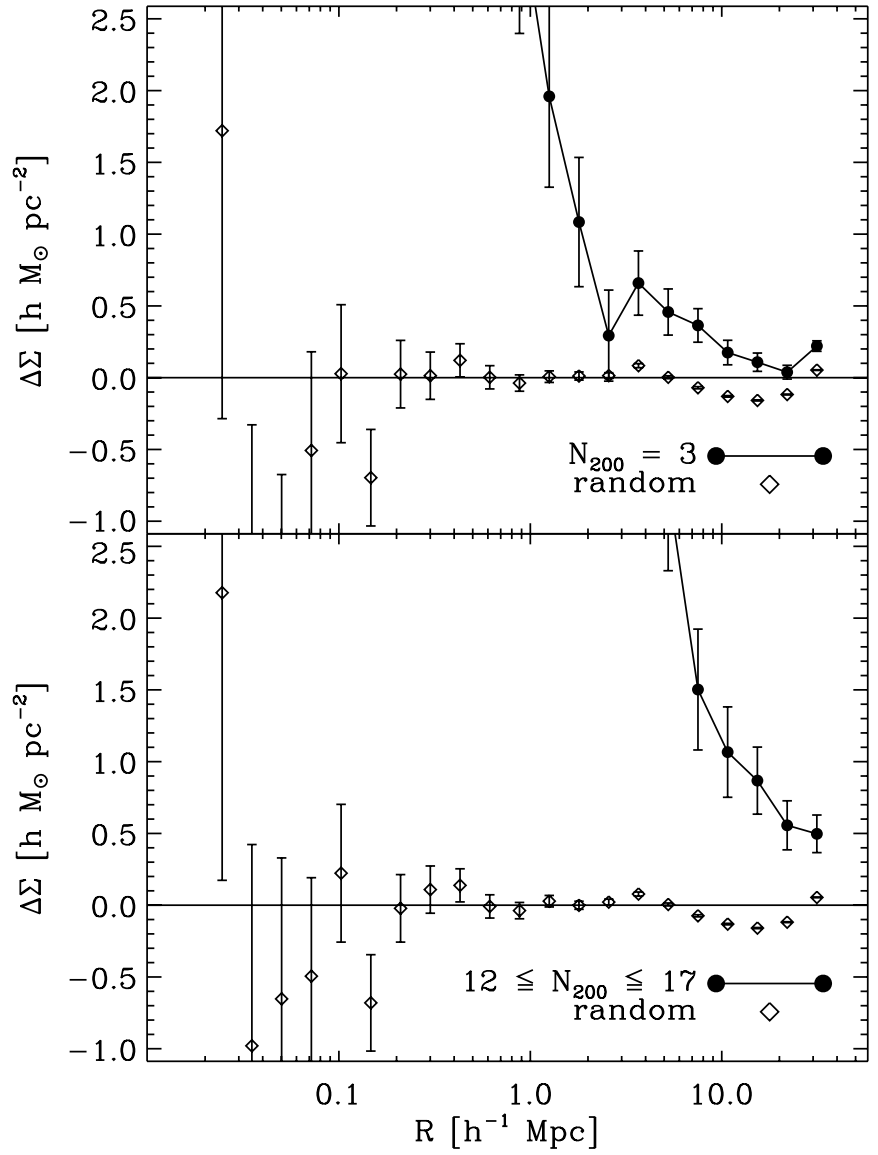

Figure 6. Lensing measurement $\Delta \Sigma$ around random points as compared to clusters. The top panel compares randoms to clusters for the $N_{200}=3$ bin; the bottom panel shows the $12 \leqslant L_{200} \leqslant 17$ bin. The nonzero detection at large scales is indicative of residual additive systematics in the PSF correction. The random signal dominates on large scales for the relatively low signal bin shown in the top panel. The contamination is less important but still significant for the intermediate bin shown in the bottom panel. The random signal is subtracted from the cluster signal for all samples presented in this work.

\subsection{Correction for Source-Lens Correlations}

The second error we correct for is the clustering of source galaxies with the lensing clusters. Although photometric redshifts help to remove cluster members from the source sample, there is still a significant contamination, especially for the richer clusters. These contribute zero to the shear, diluting the inferred profile. Because the fraction of cluster members polluting the source sample is a function of radius, this contamination alters the shape of the profile. This contamination was estimated by computing the correlation function between clusters and sources (Fischer et al. 2000; Sheldon et al. 2004). The sources are weighted exactly as in the lensing measurement. The result is shown in Figure 7 for 12 bins of the $N_{200}$ measure. In some bins, the correction factor is quite large at small scales, but is well understood.

\section{RESULTS}

The lensing $\Delta \Sigma$ profiles in 12 bins of $N_{200}$, are shown in Figure 8 . There is a significant detection for each of the bins, with $\mathrm{S} / \mathrm{N} \sim 15-20$ for all bins. The corrections described in Section 4 have been applied to each of these profiles. These data, as well as the data in Figure 11, are available for download and are summarized in Table 1.

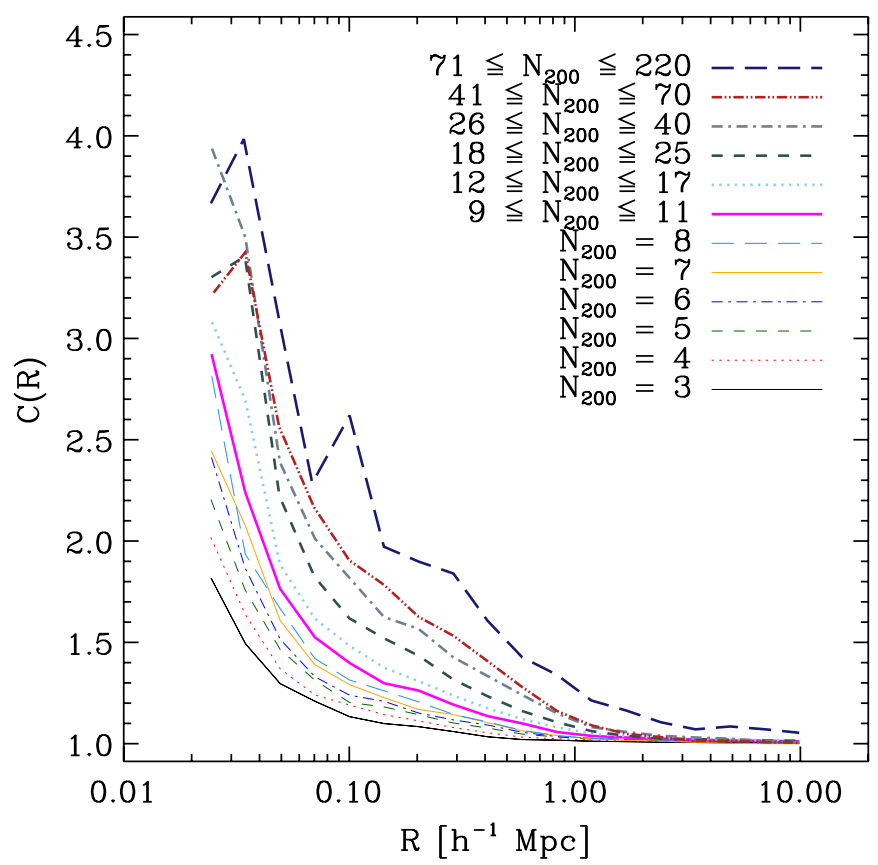

Figure 7. Correction factor for the clustering of source galaxies with the clusters in bins of $N_{200}$. The multiplicative factor $C(R)$ is calculated and applied for all profiles shown herein.

(A color version of this figure is available in the online journal.)

The errors are the diagonal elements of the covariance matrix derived from jackknife re-sampling. We use relatively small jackknife regions for this analysis, about the width of an SDSS stripe. This choice is made based on indications that variations in the systematics are the dominant source of spatial variation in the signal: the PSF also varies with roughly this scale, and of course the scans are this size. This is further supported by the fact that for much larger jackknife regions the errors are consistent with the standard Gaussian error propagation. For this jackknife, scale errors are consistent with simple error propagation for $R<1 h^{-1} \mathrm{Mpc}$, but are substantially larger on $R>5 h^{-1} \mathrm{Mpc}$ scales. The covariance matrix becomes nondiagonal for $R>5 h^{-1} \mathrm{Mpc}$, where the first off-diagonal terms are about $30 \%$. Note, at the median cluster redshift 0.25 , the width of a stripe is roughly $25 h^{-1} \mathrm{Mpc}$.

The amplitude of the lensing profile is a strong function of $N_{200}$, as expected if the number of galaxies correlates with the mass of the cluster. However, the interpretation of these profiles in terms of halo masses is complicated, as with any cluster mass measurement, by the contributions from the neighboring large-scale structure. In fact, for most of the profiles, the signal at separations larger than $2 \mathrm{Mpc}$ is entirely dominated by associated large-scale structure; this feature allows us to measure halo bias in addition to halo mass. The interpretation of these profiles in terms of halo mass and correlated mass (i.e., the halo model) is presented in detail in the companion paper Johnston et al. (2007b).

A number of the profiles in Figure 8 show deviations from a power law. Power-law fits for each bin are shown in Table 2. The fits were performed using the full covariance matrix. All but the second and fifth bins have $\chi^{2}$ per degree of freedom (dof) greater than 1.3 (dof $=19)$, indicating a poor fit. This is demonstrated visually in Figure 9, where in each case the profile has been divided by the best-fitting power law. The curves systematically have a shallower logarithmic slope at small radius and a steeper 


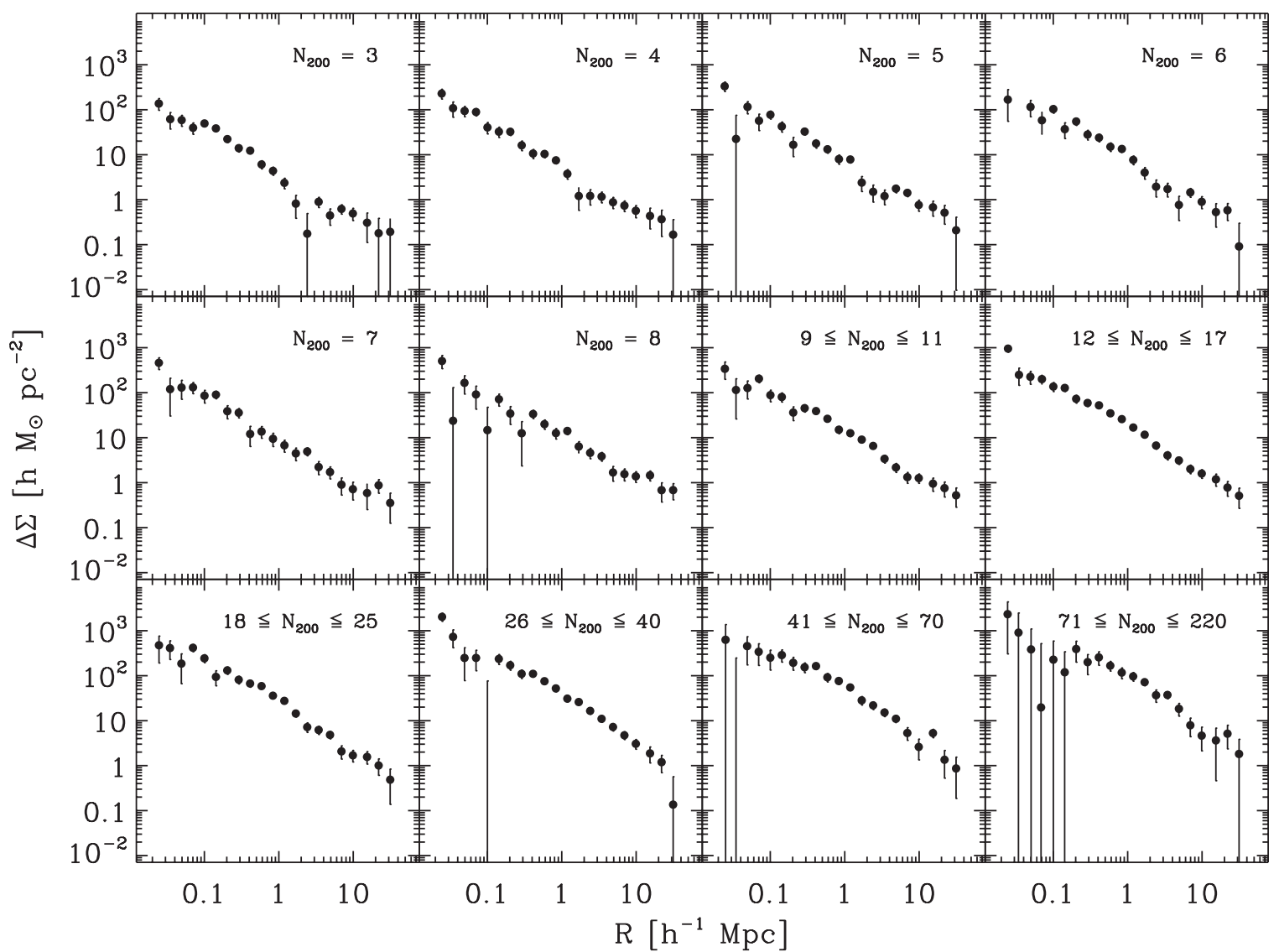

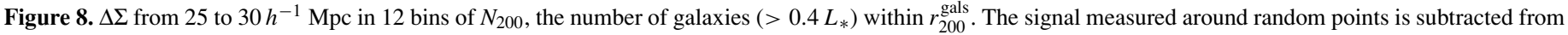
these profiles (see Figure 6). The correction for clustering of sources with the lenses is also applied (see Figure 7). The errors are from jackknife re-sampling.

slope at large radius. The shape on scales $\lesssim 100 h^{-1} \mathrm{kpc}$ may be affected by systematics, as outlined in Section 6.1. The fits are dominated by larger scales, however, and removing these points does not improve the fit significantly. There is also evidence of an upturn again at the largest radii which may be interpreted as the "two-halo term," the transition to correlations with neighboring large-scale structure. These features move to larger radius for higher $N_{200}$. The shape of these curves is in qualitative agreement with a model where the inner halo is NFW-like with a transition to linear correlations on large scales. In Johnston et al. (2007b), we demonstrate quantitatively that such a model is in fact a good fit to this data.

We split the $N_{200}$ sample further by $L_{200}$ within each bin as shown in Figure 10. In each of the $N_{200}$ bins, we split at the $2 / 3$ quantile in luminosity, such that the $2 / 3$ lowest objects are in one bin and the top $1 / 3$ are in the other. This quantile was chosen because any scaling with luminosity combined with the steep luminosity function would predict equal $\mathrm{S} / \mathrm{N}$ only for an uneven split. In the figure, the best-fitting power law has been divided out. In each case, the upper $L_{200}$ quantile has a stronger signal than the lower. Although $N_{200}$ is correlated with the lensing signal, there is additional information contained in $L_{200}$.

We explored the luminosity dependence further by splitting the clusters into 16 bins by $L_{200}$, without regard to the galaxy counts. The results of this binning are shown in Figure 11. We were able to split the sample into many more bins without losing significant precision. This is primarily due to splitting the lower $N_{200}$ bins into one or more $L_{200}$ bins. Generally the features are similar to the $N_{200}$ splits but with more dynamic range in signal
Table 1

$\Delta \Sigma$ Data for MaxBCG Clusters

\begin{tabular}{lcccc}
\hline \hline $\begin{array}{c}X \\
(\mathrm{~N} \text { or L })\end{array}$ & $\langle X\rangle$ & $\begin{array}{c}r \\
\left(h^{-1} \mathrm{Mpc}\right)\end{array}$ & $\begin{array}{c}\Delta \Sigma \\
\left(h M_{\odot} \mathrm{pc}^{-2}\right)\end{array}$ & $\begin{array}{c}\sigma(\Delta \Sigma) \\
\left(h M_{\odot} \mathrm{pc}^{-2}\right)\end{array}$ \\
\hline $\mathrm{N}$ & $3.000 \mathrm{e}+00$ & $2.438 \mathrm{e}-02$ & $1.370 \mathrm{e}+02$ & $4.000 \mathrm{e}+01$ \\
$\mathrm{~N}$ & $3.000 \mathrm{e}+00$ & $3.469 \mathrm{e}-02$ & $6.173 \mathrm{e}+01$ & $2.460 \mathrm{e}+01$ \\
$\mathrm{~N}$ & $3.000 \mathrm{e}+00$ & $4.945 \mathrm{e}-02$ & $5.881 \mathrm{e}+01$ & $1.613 \mathrm{e}+01$ \\
$\mathrm{~N}$ & $3.000 \mathrm{e}+00$ & $7.038 \mathrm{e}-02$ & $3.963 \mathrm{e}+01$ & $1.115 \mathrm{e}+01$ \\
$\mathrm{~N}$ & $3.000 \mathrm{e}+00$ & $1.003 \mathrm{e}-01$ & $4.950 \mathrm{e}+01$ & $7.657 \mathrm{e}+00$ \\
$\mathrm{~N}$ & $3.000 \mathrm{e}+00$ & $1.428 \mathrm{e}-01$ & $3.844 \mathrm{e}+01$ & $5.269 \mathrm{e}+00$ \\
$\mathrm{~N}$ & $3.000 \mathrm{e}+00$ & $2.033 \mathrm{e}-01$ & $2.224 \mathrm{e}+01$ & $3.571 \mathrm{e}+00$ \\
$\mathrm{~N}$ & $3.000 \mathrm{e}+00$ & $2.894 \mathrm{e}-01$ & $1.399 \mathrm{e}+01$ & $2.492 \mathrm{e}+00$ \\
$\mathrm{~N}$ & $3.000 \mathrm{e}+00$ & $4.120 \mathrm{e}-01$ & $1.233 \mathrm{e}+01$ & $1.839 \mathrm{e}+00$ \\
$\mathrm{~N}$ & $3.000 \mathrm{e}+00$ & $5.869 \mathrm{e}-01$ & $6.075 \mathrm{e}+00$ & $1.234 \mathrm{e}+00$ \\
$\mathrm{~N}$ & $3.000 \mathrm{e}+00$ & $8.354 \mathrm{e}-01$ & $4.373 \mathrm{e}+00$ & $8.795 \mathrm{e}-01$ \\
\hline
\end{tabular}

Notes. $\Delta \Sigma$ data corresponding to Figures 8 and 11 . The first column indicates binning on either $N_{200}$, labeled "N," or $L_{200}$, labeled "L." The second column is the mean value of $N_{200}$ or $L_{200}$ for the bin, with units of either number or $10^{10} h^{-2} L_{\odot}$.

(This table is available in its entirety in a machine-readable form in the online journal. A portion is shown here for guidance regarding its form and content.)

amplitude. We also split each $L_{200}$ bin into quantiles of $N_{200}$, but saw no significant trend.

Finally, we split the $L_{200}$ samples into bins of cluster redshift in order to quantify any redshift evolution in the signal. We split each bin at the median redshift of 0.25 . The difference relative to the errors (combined quadratically) is shown in Figure 12. No 
Table 2

Power-law Fits for $N_{200}$ Bins

\begin{tabular}{lccc}
\hline \hline \multicolumn{1}{c}{ Bin } & $A$ & $\alpha$ & $\chi^{2} / v$ \\
\hline$N_{200}=3$ & $3.2 \pm 0.2$ & $1.06 \pm 0.04$ & $51.9 / 19=2.73$ \\
$N_{200}=4$ & $4.5 \pm 0.3$ & $1.05 \pm 0.04$ & $18.1 / 19=0.951$ \\
$N_{200}=5$ & $6.4 \pm 0.4$ & $0.95 \pm 0.04$ & $41.5 / 19=2.18$ \\
$N_{200}=6$ & $7.4 \pm 0.5$ & $0.94 \pm 0.04$ & $53.4 / 19=2.81$ \\
$N_{200}=7$ & $8.1 \pm 0.7$ & $1.02 \pm 0.05$ & $19.0 / 19=1.00$ \\
$N_{200}=8$ & $10.3 \pm 0.8$ & $0.86 \pm 0.05$ & $26.7 / 19=1.41$ \\
$9 \leqslant N_{200} \leqslant 11$ & $12.5 \pm 0.6$ & $0.93 \pm 0.03$ & $26.0 / 19=1.37$ \\
$12 \leqslant N_{200} \leqslant 17$ & $16.9 \pm 0.8$ & $0.99 \pm 0.02$ & $25.1 / 19=1.32$ \\
$18 \leqslant N_{200} \leqslant 25$ & $23 \pm 1$ & $0.99 \pm 0.03$ & $38.9 / 19=2.05$ \\
$26 \leqslant N_{200} \leqslant 40$ & $34 \pm 2$ & $0.95 \pm 0.02$ & $49.3 / 19=2.59$ \\
$41 \leqslant N_{200} \leqslant 70$ & $47 \pm 2$ & $0.89 \pm 0.03$ & $48.9 / 19=2.57$ \\
$71 \leqslant N_{200} \leqslant 220$ & $77 \pm 7$ & $0.91 \pm 0.04$ & $28.1 / 19=1.48$ \\
\hline
\end{tabular}

Notes. Power-law fits for each bin in $N_{200}: \Delta \Sigma=A R^{-\alpha}$ with $R$ in units of $h^{-1} \mathrm{Mpc}$ and $A$ in units of $h M_{\odot} \mathrm{pc}^{-2}$. The reduced $\chi^{2}$ is relatively high in most bins, indicating a power law is not a good fit. There is a strong correlation between the power-law amplitude $A$ and richness $N_{200}$, while the best-fit $\alpha$ is relatively stable.

evolution is evident. The distribution of $\chi^{2}$ between the $16 L_{200}$ bins is consistent with that expected from random deviations for 21 dof. We also averaged the signal across all luminosities and found no evidence for evolution. Although not presented here for the sake of brevity, we also have a null detection of redshift evolution in bins of $N_{200}$.

\section{FURTHER SYSTEMATIC EFFECTS}

We have corrected the lensing measurements for additive errors and the clustering of sources with lenses (see Section 4). In this section, we shall discuss other possible sources of systematic error. Many of these issues have been addressed in detail in Mandelbaum et al. (2005). We will briefly comment here on a few of the more important issues, in particular the photometric redshifts for which our analysis differs from that study, and intrinsic alignments in clusters.

\subsection{Lensing Measurement Errors}

There are two basic types of errors on lensing measurements: multiplicative (calibration) errors and additive errors. We described the corrections applied for additive errors in Section 4.

Calibration errors in $\Delta \Sigma$ come in two types. Recall the definition of $\Delta \Sigma$ :

$$
\Delta \Sigma(R)=\gamma_{T}(R) \times \Sigma_{\text {crit }}
$$

Errors may occur in converting the measured galaxy shapes to shear $\gamma_{T}$, or in measuring the critical density $\Sigma_{\text {crit }}$, which depends on the angular diameter distances to lens and source.

Errors in the shear calibrations may be due to residuals in the PSF dilution correction or incorrect shape to shear transformations. The shape to shear transformation is measured directly from the data (see Section 2). It is determined much better than our signal, so it should be a minimal effect. The dilution corrections discussed in Section 3.3, however, can only be determined in a model-dependent way. Thus, it is difficult to empirically determine the accuracy of the correction. As a further test, we split the source sample into bins of size and saw no variation of the recovered the signal within the errors. This, however, does not demonstrate that there is no overall calibration error.

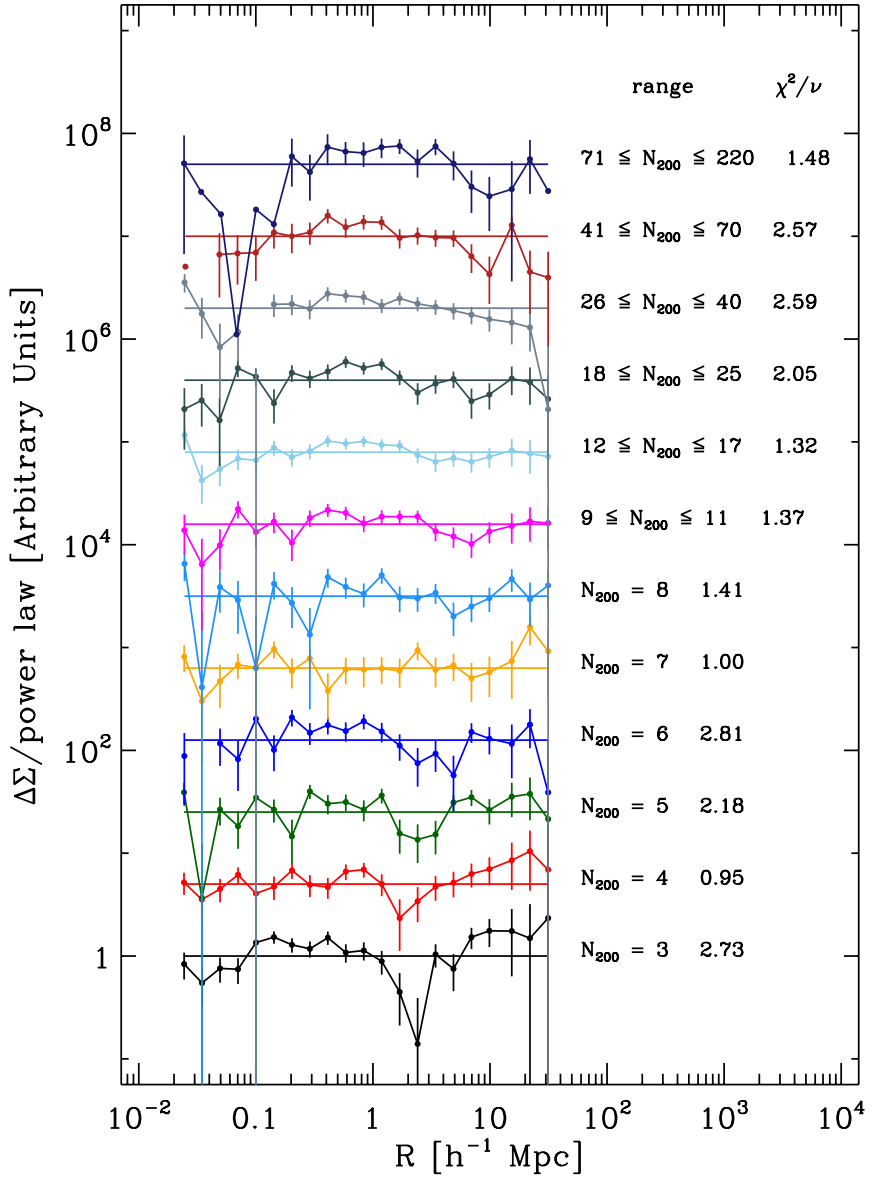

Figure 9. Power-law fits in each $N_{200}$ bin. For each bin, the best-fitting power law (see Table 2) has been divided out and the signal scaled arbitrarily to separate the profiles visually. For clarity, the error bars for points with $\mathrm{S} / \mathrm{N}<1$ have been suppressed for the top two bins. Each curve is labeled by its richness range and reduced $\chi^{2}$ for the power-law fit. The profiles are not generally good fits to a power law and demonstrate systematic deviations as a function of scale.

(A color version of this figure is available in the online journal.)

The calibrations were tested using simulations in the second Shear Testing Programme, also known as STEP2 (Massey et al. 2007). For the PSFs tested therein, the calibration was recovered to a few percent accuracy, but there is an inherent uncertainty when any such scheme is applied to real data. In particular, the PSF in the STEP2 images are different from the typical SDSS PSF.

The PSF is not constant, but varies in time and position in the focal plane. This variation is mapped by the measurement of a finite number of stars per field. The algorithm used by the SDSS to model the PSF (Lupton et al. 2001; Sheldon et al. 2004), while quite powerful, is inevitably limited in the spatial frequency with which it can map changes in the PSF. The density of the stars used is about one per 6.7 square arcmin, or a spacing of 2.6 arcmin. Any PSF variations on scales smaller than this are simply not accounted for.

A recent study by Jarvis \& Jain (2004) has shown that this limitation can be overcome if the PSF patterns are recurring and depend on only a few major variables, such as position in the focal plane and focus. We will explore this method in future work.

The errors in estimating $\Sigma_{\text {crit }}$ come from errors in the estimating the photometric redshifts. We estimated these error distributions for our sample and include them in our calculations (see Section 3.4). The essential assumption in determining these 


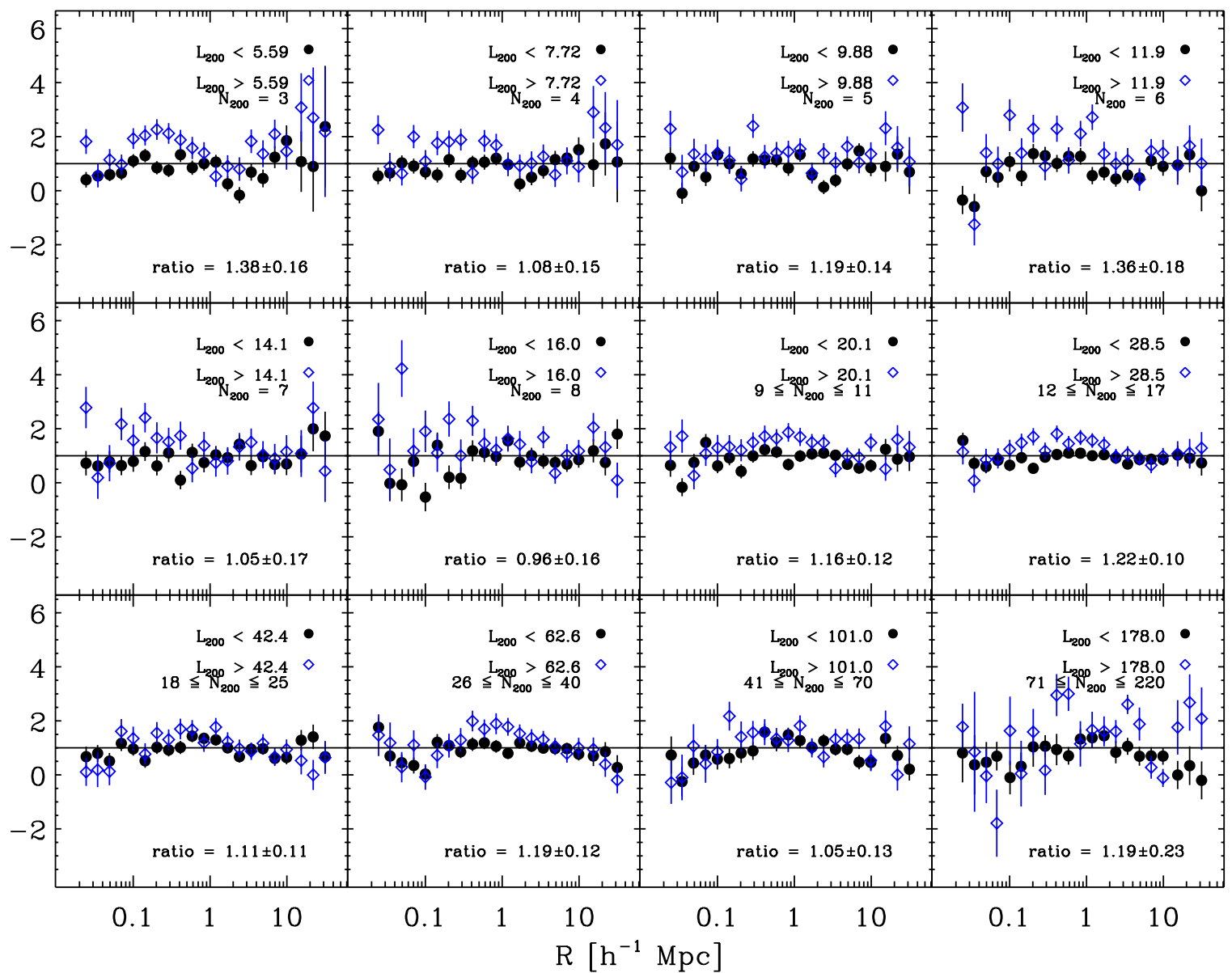

Figure 10. Same $12 N_{200}$ bins from Figure 8 with each bin split into two by $L_{200}$. The $L_{200}$ split is at the $2 / 3$ quantile. The upper quantile is represented by the diamond symbols, the lower by the filled circles. The best-fitting power law for each $N_{200}$ bin before splitting is divided out. The mean ratio of the splits is shown in the legend. There is a further correlation with luminosity within each $N_{200}$ bin.

(A color version of this figure is available in the online journal.)

biases is that the five band magnitudes of the SDSS is sufficient information to determine the redshift distribution of a given set of objects. If this is not true, for example if surface brightness or size matter and the training set differs from the photometric set in this regard, the photo- $z$ 's may be biased. This was tested in Mandelbaum et al. (2005) by using a sample of luminous red galaxies for which a well matched training sample can be used, and consistency was found at the $10 \%$ level. We have not repeated that experiment for this data, but expect the same level of consistency.

On small scales the sources may be mismeasured due to the presence of the brightest cluster galaxy, on which we center our lensing measurements. In two SDSS studies (Masjedi et al. 2006; Mandelbaum et al. 2006a), it was shown that on $\lesssim 20^{\prime \prime}$ scales the presence of bright LRGs will bias the photometry and size measurement of neighboring galaxies significantly. This is essentially a calibration error because it may cause errors in the photometric redshifts and dilution correction. Because the BCGs are similar to LRGs, both in intrinsic properties and redshift distribution, similar effects are expected to impact this study. We have not, however, repeated these tests for the current sample. For this reason, we do not correct for such effects, but rather caution the reader that, for scales $\lesssim 50 h^{-1} \mathrm{kpc}$, the profiles presented in this work may be biased. For mass studies at the virial radius this is an insignificant volume and can be safely ignored, but caution should be used for studies of the inner profile.

\subsection{Intrinsic Alignments}

We assume that the measured correlations between the shapes of the source galaxies are due to gravitational lensing. In practice, the shapes of galaxies themselves may be correlated. In general, this mostly washes out in a measurement such as ours as long as the sources are far behind the lens. This is because we correlate with the tangential frame around lenses and the sources are at a large range of distances. In practice, however, it is difficult to identify a sample of sources which are all truly separate from the lenses, so if intrinsic alignments exist the signal may indeed be contaminated. Limits from data (see Hirata et al. 2004, and references therein) indicate that this is a small $(<15 \%)$ contaminant to the tangential shear for galaxygalaxy lensing surveys. The contamination may be higher for clusters because a larger number of source galaxies are actually physically associated with the lens.

In order to test the effects of alignments, we used clusters determined from SDSS spectroscopy as presented in Berlind et al. (2006). We used the volume-limited sample with absolute magnitude in the $r$ band less than -20 . There are 4119 clusters in this sample. For tracers of the intrinsic alignment, we use all galaxies from the SDSS "main" spectroscopic galaxy sample over the same regions; there is no need for a volumelimited sample of shape tracers. See Strauss et al. (2002) for a description of the target selection algorithm. The use of only spectroscopic redshifts for clusters and tracers greatly reduces 


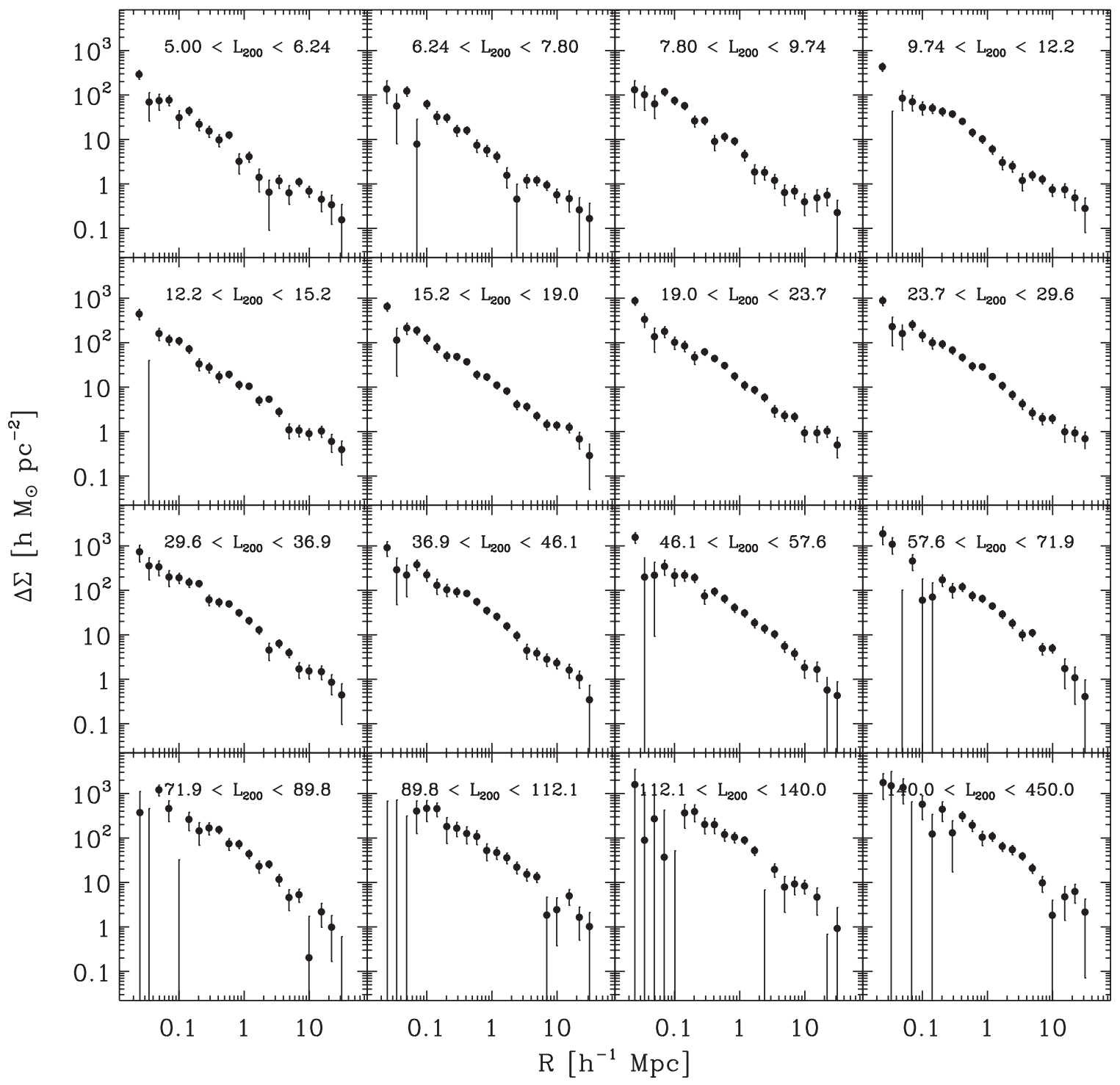

Figure 11. Same as Figure 8 but in 16 bins of $L_{200}$, the total $i$-band luminosity of ( $>0.4 L_{*}$ ) galaxies within $r_{200}^{\text {gals }}$.

the fraction of physically unassociated pairs. We chose all galaxies within our search aperture and with velocities within $\pm 2000 \mathrm{~km} \mathrm{~s}^{-1}$. No attempt was made to match the luminosity distribution of the tracers to those of our actual source galaxies. Shapes were corrected for PSF effects using the same techniques described in Section 4.

Figure 13 shows the mean tangential intrinsic shear measured for these clusters. There is no detected signal; we place only limits on the effect. For example, the intrinsic shear within $100 h^{-1} \mathrm{kpc}$ is $-0.0058<\left\langle\gamma_{T}\right\rangle<0.0025$ at $95 \%$ confidence.

In order to estimate the limit of contamination in our data we took the limits of this shear and scaled them to $\Delta \Sigma$ for each of our lens samples given their mean redshift. We also multiplied by the fraction of source galaxies that were actually in the clusters, and the boost factor for clustering of sources with the lenses. This last factor is exactly that is shown in Figure 7. The mean-estimated contamination is shown in Figure 14 for each of the $N_{200}$ bins. This plot is cumulative, so adjacent points are correlated. Table 3 shows limits on $\Delta \Sigma$ in each of the $N_{200}$ bins within $100 h^{-1} \mathrm{kpc}$. These limits are not very stringent but are all less than the mean signal in absolute value.
The Berlind et al. (2006) clusters are not selected in the same fashion as the MaxBCG sample, so there is additional uncertainty in applying these limits. Any sample selected to have spectroscopy will be selected differently, and will be relatively few in number; this is a limit of the current observations. Also, the effect may depend on richness, a possibility we are unable to address due to the relatively small sample of Berlind et al. (2006).

As a further test of the effect of intrinsic alignments, we required the source photo- $z$ in the MaxBCG shear measurements to be at least $z$ (cluster) +0.2 , but saw no change in the shear signal. This could also mean that the redshifts are dominated by random noise at the level of 0.2 , but the mean error at these magnitudes is expected to be $\lesssim 0.05$ (see Section 3.4)

\section{COMPLICATIONS FOR INTERPRETATION}

There are a few effects that may complicate the interpretation of these results. Firstly, the shear in the inner portions of the largest galaxy clusters is not weak so interpretation of shear in terms of $\Delta \Sigma$ is incorrect. This is important for the largest 


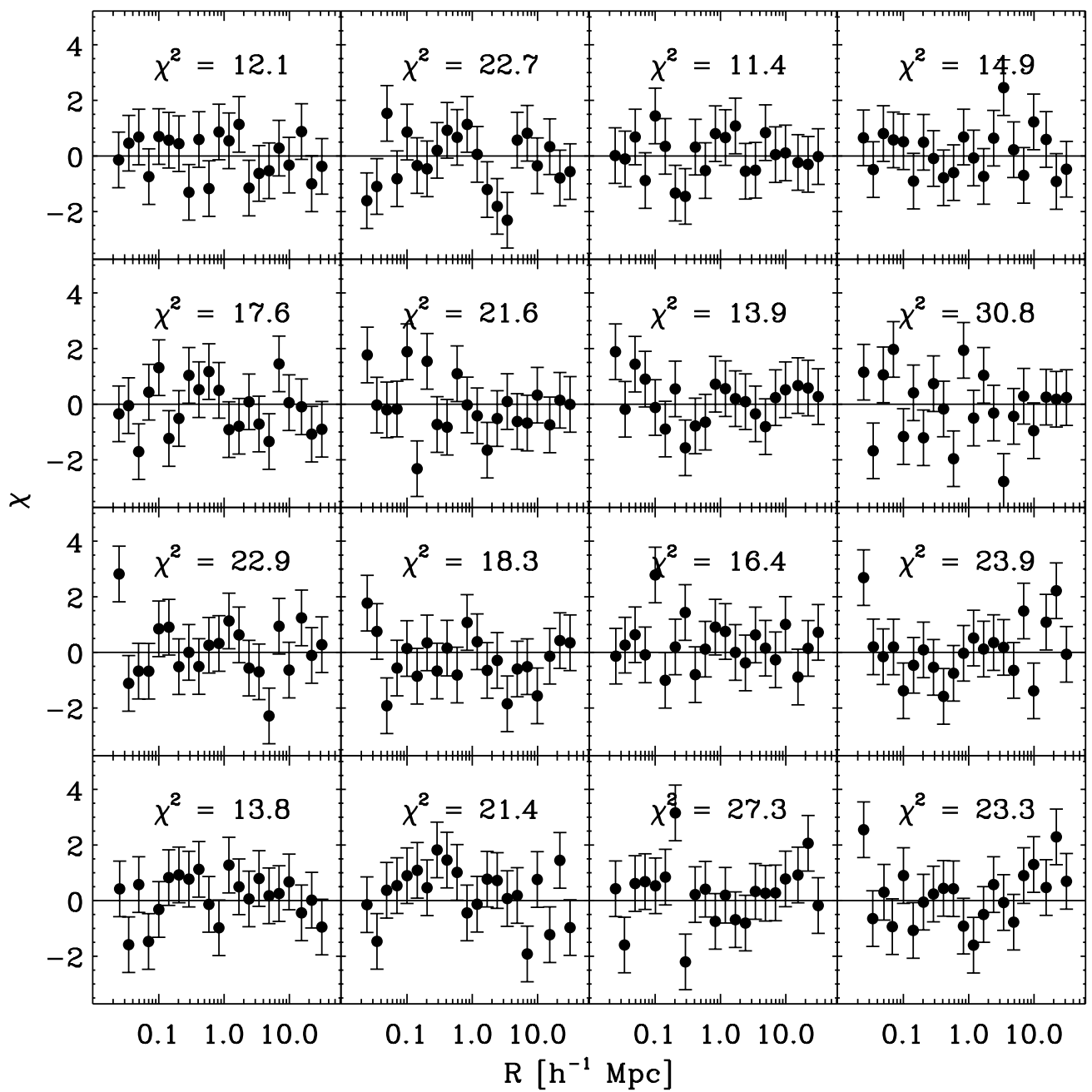

Figure 12. Bins of luminosity as in Figure 11 with each $L_{200}$ bin split at the median redshift of 0.25 . The data points are the difference between high-redshift and low-redshift splits divided by the error (added quadratically). The distribution of $\chi^{2}$ between the 16 bins is consistent with that expected from random scatter with 21 dof. The mean over all luminosities is also consistent with no evolution.

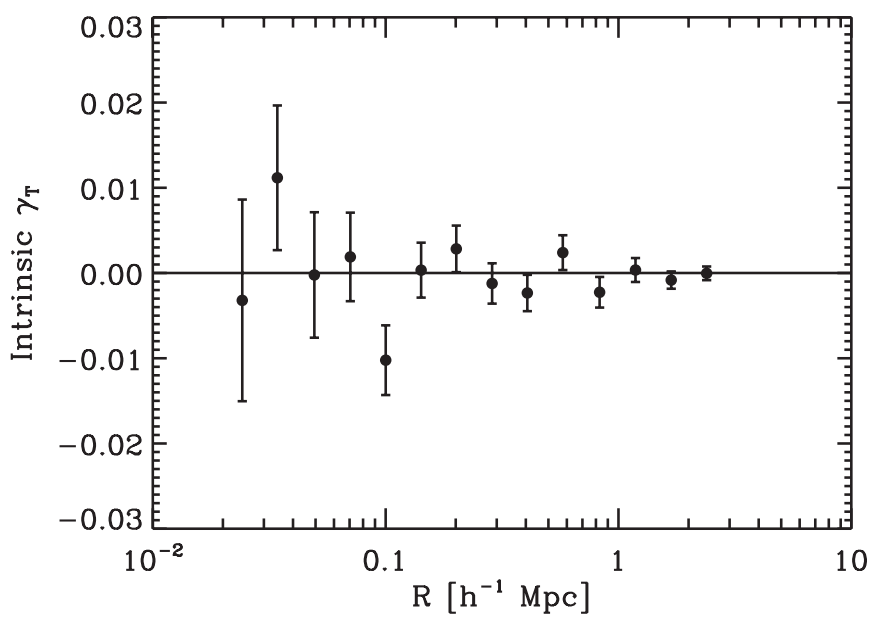

Figure 13. Estimate of intrinsic tangential alignments for the spectroscopic cluster sample of Berlind et al. (2006). The shear tracer population was drawn from the SDSS main spectroscopic sample and chosen to have velocity within $\pm 2000 \mathrm{~km} \mathrm{~s}^{-1}$ of the cluster. Thus any signal is not due to lensing, but rather intrinsic correlations between galaxy shapes and the tangential direction relative to the lens center. Such an effect would bias the lensing profiles. No net effect was detected. clusters on $\lesssim 100 h^{-1} \mathrm{kpc}$ scales. This may be accounted for in a straightforward way when modeling the signal (Mandelbaum et al. 2006a; Johnston et al. 2007b). Second, the cluster center, chosen as the location of the BCG, may not correspond to the center of mass. This acts like a convolution of the profile with the distribution of BCG offsets. Again, this is only important on relatively small scales. We will leave these issues for the follow-up paper Johnston et al. (2007b).

A third issue is that of the richness measure $N_{200}$. There is a statistical relationship between the number of galaxies counted by the MaxBCG cluster finder $N_{200}$ and the true number of galaxies in the halo. This is partly due to the fact that we observe the cluster in projection, so galaxies of the right type and color but outside the cluster in the neighboring large-scale structure are counted. It is also partly due to contamination of the counts due to degeneracies in the space of galaxy type and redshift. In this paper and in the follow-up paper Johnston et al. (2007b) we choose, for simplicity, to compare the lensing signal to the basic observable $N_{200}$, and do not attempt to account for the statistical relation between the measured counts and the true counts. In order to further interpret the measurements in terms of physical models this relationship must be taken into account. Rozo et al. (2009) model this effect in their cosmological analysis of the MaxBCG. 

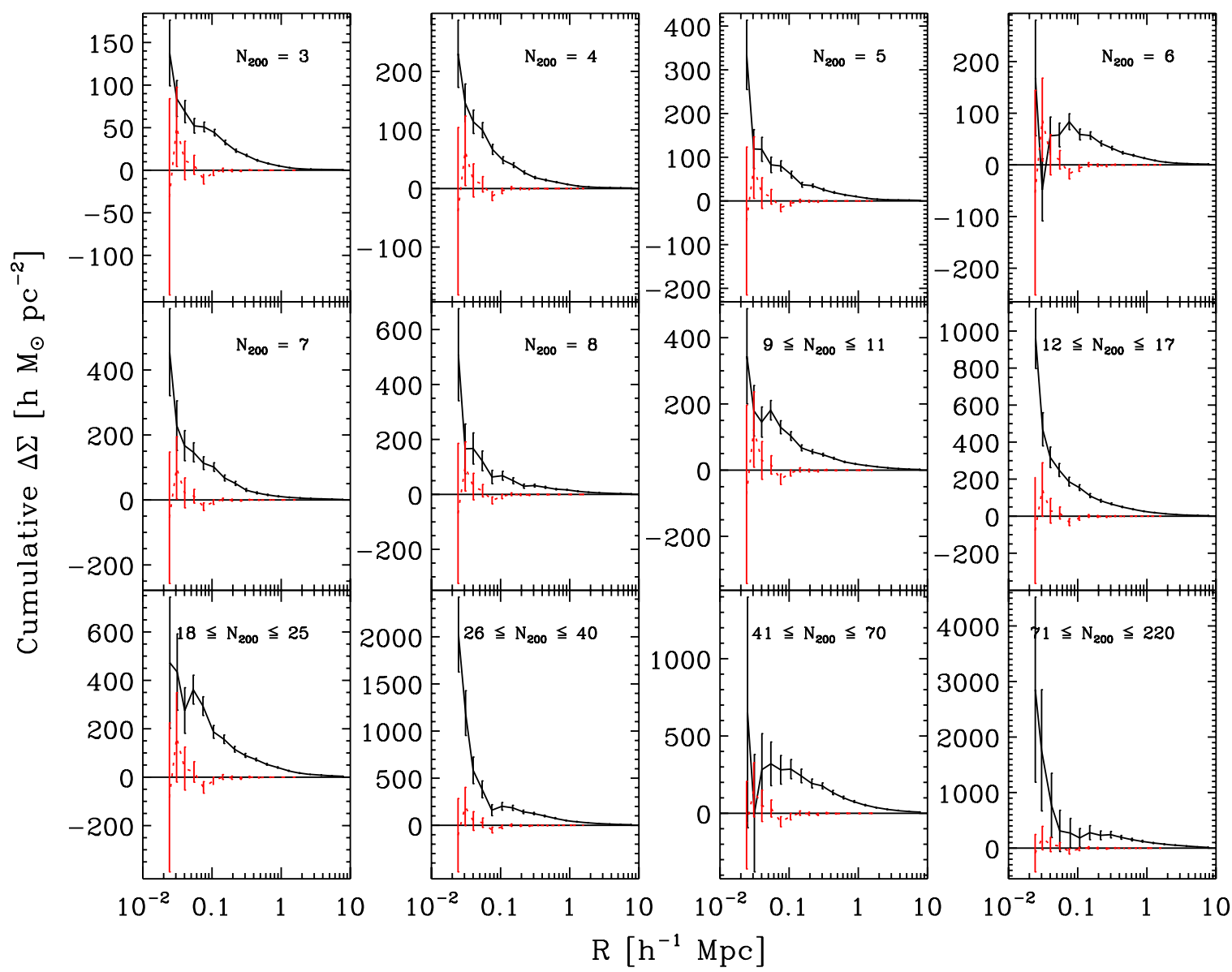

Figure 14. Mean-estimated contamination of $\Delta \Sigma$ from intrinsic alignments in each of the $N_{200}$ bins, estimated as described in the text. These are cumulative curves so adjacent bins are correlated. In each panel, the intrinsic shear is the red curve and the cluster measurement is the black curve. Note the vertical scale varies between plots.

(A color version of this figure is available in the online journal.)

\section{SUMMARY}

We have measured ensemble lensing due to clusters of galaxies over scales $20 h^{-1} \mathrm{kpc}$ to $30 h^{-1} \mathrm{Mpc}$. We split the sample into 12 independent bins of richness $N_{200}$ and 16 bins of $i$-band luminosity $L_{200}$, with strong detections $(\mathrm{S} / \mathrm{N} \sim 15-20)$ in each bin. The profiles were corrected for systematic effects, including additive shear errors and clustering of sources with the lens clusters. We placed limits on the amount of contamination in our signal due to intrinsic alignments and concluded that on $100 \mathrm{kpc}$ scales and greater the effect is not dominant; the limits on smaller scales are weak. Calibration errors are less well known. These calibration errors are most likely dominated by uncertainties in determining the redshift distribution of the sources, which are expected to be of order $10 \%$; the shear calibration errors are expected to be a few percent. The shape of these profiles, and relative scaling, are insensitive to calibration uncertainties.

Interpretation of these profiles on small scales requires some caution. For the most massive clusters, the lensing effect on $\lesssim 100 h^{-1} \mathrm{kpc}$ scales is most likely nonlinear and this must be accounted for in models. Furthermore, on $\lesssim 50 h^{-1} \mathrm{kpc}$ (the first two bins) there may be significant systematic effects in the photometry and size measurements of source galaxies due to the extended light profile of the brightest cluster galaxy. This effect has been seen around bright galaxies in the SDSS in other studies (Masjedi et al. 2006; Mandelbaum et al. 2006a). Because
Table 3

Intrinsic Alignment Limits for $N_{200}$ Bins

\begin{tabular}{lcc}
\hline \hline \multicolumn{1}{c}{ Bin } & $\Delta \Sigma_{\text {int }}$ Limits $(95 \%)$ & $\langle\Delta \Sigma\rangle$ \\
\hline$N_{200}=3$ & {$[-15.0,4.03]$} & $39.6 \pm 4.05$ \\
$N_{200}=4$ & {$[-20.1,5.93]$} & $46.5 \pm 6.09$ \\
$N_{200}=5$ & {$[-24.3,6.12]$} & $56.8 \pm 8.30$ \\
$N_{200}=6$ & {$[-27.9,6.78]$} & $48.1 \pm 10.9$ \\
$N_{200}=7$ & {$[-30.8,8.26]$} & $73.0 \pm 13.6$ \\
$N_{200}=8$ & {$[-34.3,9.28]$} & $61.0 \pm 16.9$ \\
$9 \leqslant N_{200} \leqslant 11$ & {$[-39.8,11.3]$} & $91.4 \pm 13.6$ \\
$12 \leqslant N_{200} \leqslant 17$ & {$[-49.4,14.2]$} & $137 \pm 16.4$ \\
$18 \leqslant N_{200} \leqslant 25$ & {$[-65.5,17.8]$} & $157 \pm 27.4$ \\
$26 \leqslant N_{200} \leqslant 40$ & {$[-73.5,23.0]$} & $207 \pm 41.9$ \\
$41 \leqslant N_{200} \leqslant 70$ & {$[-91.7,26.9]$} & $257 \pm 68.8$ \\
$71 \leqslant N_{200} \leqslant 220$ & {$[-120,53.9]$} & $232 \pm 184$ \\
\hline
\end{tabular}

Notes. Limits on the contamination of $\Delta \Sigma$ from intrinsic alignments for each $N_{200}$ bin within a radius of $100 h^{-1} \mathrm{kpc}$. The limits are shown as $95 \%$ confidence intervals. For comparison, the mean $\Delta \Sigma$ for clusters within the same radius is listed in the last column.

the relative volume interior to these radii is small, virial mass estimates should be robust, but a study of the inner profiles will require further characterization of these effects. Neither of these issues should be important for scales $\gtrsim 100 \mathrm{~h}^{-1} \mathrm{kpc}$. On the other hand, the BCG location chosen as the center may be offset from the true mass peak and this can have effects to larger radius (Johnston et al. 2007b). 
The signal is dependent on richness and luminosity on all scales. We fit power-law models and found the amplitude is a strong function of $N_{200}$, while the power-law index is relatively insensitive to richness. However, the signal is a poor fit to a power law in most richness bins and the deviations from a power law are systematic. The logarithmic slope generally runs from shallower to steeper with increasing radius. We will interpret these curves in terms of a more appropriate model with a universal halo profile and linear correlations on large scales in Johnston et al. (2007b).

Because the number of galaxies $N_{200}$ is not directly related to mass or luminosity, one may expect a broad spread in luminosity and mass for a given $N_{200}$ bin. We explored this by splitting each $N_{200}$ bin into quantiles of luminosity $L_{200}$. We found a scaling of the signal with $L_{200}$ within each $N_{200}$ bin, indicating that there is significant mass scatter in $N_{200}$ bins, and that mass may scale more strongly with $L_{200}$ than $N_{200}$.

Finally, we explored the dependence of the signal on redshift by splitting each of the luminosity bins at the mean redshift 0.25 . We detect no evolution within our uncertainties for the current sample, though the redshift range $0.1<z<0.3$ is relatively small.

The precision of these measurements is sufficient to perform nonparametric inversions to the mean three-dimensional mass density. In the companion paper Johnston et al. (2007b), we present these inversions in each bin of richness and luminosity. We then infer the model-independent virial mass and large-scale bias. We also interpret these profiles in terms of a universal halo profile on small scales and linear bias on large scales. In Sheldon et al. (2007), we combine the nonparametric mass profiles from Johnston et al. (2007b) with nonparametric light profiles to measure the mean mass-to-light ratios around the MaxBCG clusters. In Rozo et al. (2009), we use the massobservable relation from Johnston et al. (2007b) to constrain the mass function of halos and cosmological parameters.

Thanks to David Hogg and Michael Blanton for so many useful discussions, and to Roman Scoccimarro and Mulin Ding for use of the "Mafalda" computing cluster. Many thanks to the anonymous referee for excellent comments and constructive criticism.

E.S.S. was supported in part by NSF grant AST-0428465 and the U.S. Department of Energy under contract No. DEAC02-98CH10886. B.P.K. and T.A.M. gratefully acknowledge support from NSF grant AST 044327 and the Michigan Center for Theoretical Physics. D.E.J. received partial support from the U.S. Department of Energy under contract number DE-FG0208ER41567. The research described in this paper was performed in part at the Jet Propulsion Laboratory, California Institute of Technology, under a contract with the National Aeronautics and Space Administration.

Funding for the SDSS and SDSS-II has been provided by the Alfred P. Sloan Foundation, the ParticipatingInstitutions, the National Science Foundation, the U.S. Department of Energy, the National Aeronautics and Space Administration, the Japanese Monbukagakusho, the Max Planck Society, and the Higher Education Funding Council for England. The SDSS Web site is http://www.sdss.org/.

The SDSS is managed by the Astrophysical Research Consortium for the Participating Institutions. The Participating Institutions are the American Museum of Natural History, Astrophysical Institute Potsdam, University of Basel, Cambridge University, Case Western Reserve University, Univer- sity of Chicago, Drexel University, Fermilab, the Institute for Advanced Study, the Japan Participation Group, Johns Hopkins University, the Joint Institute for Nuclear Astrophysics, the Kavli Institute for Particle Astrophysics and Cosmology, the Korean Scientist Group, the Chinese Academy of Sciences (LAMOST), Los Alamos National Laboratory, the Max-PlanckInstitute for Astronomy (MPIA), the Max-Planck-Institute for Astrophysics (MPA), New Mexico State University, Ohio State University, University of Pittsburgh, University of Portsmouth, Princeton University, the United States Naval Observatory, and the University of Washington.

\section{REFERENCES}

Adelman-McCarthy, J. K., et al. 2006, ApJS, 162, 38

Becker, M. R., et al. 2007, ApJ, 669, 905

Berlind, A. A., et al. (The SDSS Collaboration) 2006, ApJS, 167, 1

Bernstein, G. M., \& Jarvis, M. 2002, AJ, 123, 583

Böhringer, H., et al. 2001, A\&A, 369, 826

Carlberg, R. G., Yee, H. K. C., Ellingson, E., Abraham, R., Gravel, P., Morris, S., \& Pritchet, C. J. 1996, ApJ, 462, 32

Clowe, D., Bradač, M., Gonzalez, A. H., Markevitch, M., Randall, S. W., Jones, C., \& Zaritsky, D. 2006, ApJ, 648, L109

Clowe, D., Luppino, G. A., Kaiser, N., \& Gioia, I. M. 2000, ApJ, 539, 540

Collins, C. A., et al. 2000, MNRAS, 319, 939

Collister, A. A., \& Lahav, O. 2004, PASP, 116, 345

Dahle, H., Kaiser, N., Irgens, R. J., Lilje, P. B., \& Maddox, S. J. 2002, ApJS, 139,313

Ebeling, H., Edge, A. C., Bohringer, H., Allen, S. W., Crawford, C. S., Fabian, A. C., Voges, W., \& Huchra, J. P. 1998, MNRAS, 301, 881

Eisenstein, D. J., et al. 2001, AJ, 122, 2267

Fahlman, G., Kaiser, N., Squires, G., \& Woods, D. 1994, ApJ, 437, 56

Fischer, P., \& Tyson, J. A. 1997, AJ, 114, 14

Fischer, P., et al. 2000, AJ, 120, 1198

Fukugita, M., et al. 1996, AJ, 111, 1748

Gao, L., White, S. D. M., Jenkins, A., Stoehr, F., \& Springel, V. 2004, MNRAS, 355,819

Gott, J. R. I., Jurić, M., Schlegel, D., Hoyle, F., Vogeley, M., Tegmark, M., Bahcall, N., \& Brinkmann, J. 2005, ApJ, 624, 463

Gunn, J. E., et al. 1998, AJ, 116, 3040

Gunn, J. E., et al. 2006, AJ, 131, 2332

Hansen, S. M., McKay, T. A., Wechsler, R. H., Annis, J., Sheldon, E. S., \& Kimball, A. 2005, ApJ, 633, 122

Henry, J. P., Gioia, I. M., Maccacaro, T., Morris, S. L., Stocke, J. T., \& Wolter, A. $1992, \mathrm{ApJ}, 386,408$

Hirata, C., \& Seljak, U. 2003, MNRAS, 343, 459

Hirata, C. M., et al. 2004, MNRAS, 353, 529

Hoekstra, H. 2003, MNRAS, 339, 1155

Hoekstra, H., Franx, M., Kuijken, K., \& Squires, G. 1998, ApJ, 504, 636

Hoekstra, H., et al. 2001, ApJ, 548, L5

Hogg, D. W., Finkbeiner, D. P., Schlegel, D. J., \& Gunn, J. E. 2001, AJ, 122, 2129

Ivezić, Ž., et al. 2004, Astron. Nachr., 325, 583

Jarvis, M., \& Jain, B. 2004, arXiv:astro-ph/0412234

Jenkins, A., Frenk, C. S., White, S. D. M., Colberg, J. M., Cole, S., Evrard, A. E., Couchman, H. M. P., \& Yoshida, N. 2001, MNRAS, 321, 372

Joffre, M., et al. 2000, ApJ, 534, L131

Johnston, D. E., et al. 2007a, ApJ, 656, 27

Johnston, D. E., et al. 2007b, arXiv:0709.1159

Katgert, P., Biviano, A., \& Mazure, A. 2004, ApJ, 600, 657

Koester, B. P., et al. 2007a, ApJ, 660, 239

Koester, B. P., et al. 2007b, ApJ, 660, 221

Kravtsov, A. V., Berlind, A. A., Wechsler, R. H., Klypin, A. A., Gottlöber, S., Allgood, B., \& Primack, J. R. 2004, ApJ, 609, 35

Lilly, S. J., Le Fevre, O., Crampton, D., Hammer, F., \& Tresse, L. 1995, ApJ, 455,50

Lima, M., Cunha, C. E., Oyaizu, H., Frieman, J., Lin, H., \& Sheldon, E. S. 2008, MNRAS, 390, 118

Luppino, G. A., \& Kaiser, N. 1997, ApJ, 475, 20

Lupton, R. H., et al. 2001, in ASP Conf. Ser. 238, Astronomical Data Analysis Software and Systems X, ed. F. R. Harnden, Jr., F A. Primini, \& H. E. Payne (San Francisco, CA: ASP), 269

Mahdavi, A., \& Geller, M. J. 2004, ApJ, 607, 202 
Mandelbaum, R., Seljak, U., Cool, R. J., Blanton, M., Hirata, C. M., \& Brinkmann, J. 2006a, MNRAS, 372, 758

Mandelbaum, R., Seljak, U., Kauffmann, G., Hirata, C. M., \& Brinkmann, J. 2006b, MNRAS, 368, 715

Mandelbaum, R., et al. 2005, MNRAS, 361, 1287

Mao, S., Jing, Y., Ostriker, J. P., \& Weller, J. 2004, ApJ, 604, L5

Masjedi, M., et al. 2006, ApJ, 644, 54

Massey, R., et al. 2007, MNRAS, 376, 13

Metzler, C. A., White, M., \& Loken, C. 2001, ApJ, 547, 560

Mo, H. J., \& White, S. D. M. 1996, MNRAS, 282, 347

Navarro, J. F., Frenk, C. S., \& White, S. D. M. 1997, ApJ, 490, 493

Neumann, D. M., Lumb, D. H., Pratt, G. W., \& Briel, U. G. 2003, A\&A, 400 811

Oyaizu, H., Lima, M., Cunha, C. E., Lin, H., Frieman, J., \& Sheldon, E. S. 2008, ApJ, 674, 768

Parker, L. C., Hudson, M. J., Carlberg, R. G., \& Hoekstra, H. 2005, ApJ, 634, 806

Pier, J. R., et al. 2003, AJ, 125, 1559

Press, W. H., \& Schechter, P. 1974, ApJ, 187, 425

Reiprich, T. H., \& Böhringer, H. 2002, ApJ, 567, 716

Rozo, E., et al. 2009, ApJ, in press (arXiv:0902.3702)
Schlegel, D. J., Finkbeiner, D. P., \& Davis, M. 1998, ApJ, 500, 525

Scranton, R., et al. 2002, ApJ, 579, 48

Scranton, R., et al. 2003, arXiv:astro-ph/0307335

Scranton, R., et al. 2005, ApJ, 633, 589

Seljak, U., \& Warren, M. S. 2004, MNRAS, 355, 129

Sheldon, E. S., et al. 2001, ApJ, 554, 881

Sheldon, E. S., et al. 2004, AJ, 127, 2544

Sheldon, E. S., et al. 2009, ApJ, 703, 2232

Sheth, R. K., \& Tormen, G. 1999, MNRAS, 308, 119

Smith, J. A., et al. 2002, AJ, 123, 2121

Stoughton, C., et al. 2002, AJ, 123, 485

Strauss, M. A., et al. 2002, AJ, 124, 1810

Tucker, D. L., et al. 2006, Astron. Nachr., 327, 821

Tyson, J. A., \& Fischer, P. 1995, ApJ, 446, L55

Tyson, J. A., Wenk, R. A., \& Valdes, F. 1990, ApJ, 349, L1

Umetsu, K., Broadhurst, T., Takada, M., \& Kong, X. 2005, J. Korean Astron. Soc., 38, 191

White, M., van Waerbeke, L., \& Mackey, J. 2002, ApJ, 575, 640

Wittman, D., Margoniner, V. E., Tyson, J. A., Cohen, J. G., Becker, A. C., \& Dell'Antonio, I. P. 2003, ApJ, 597, 218

Yee, H. K. C., et al. 2001, VizieR Online Data Catalog, 212, 90475

York, D. G., et al. 2000, AJ, 120, 1579 